\title{
Deformable Registration for Image-Guided Ra- diation Therapy
}

\author{
David Sarrut ${ }^{\mathrm{PhD}, 1,2}$,
}

Address 1: Radiotherapy department, Centre Léon Bérard, 28 rue Laënnec, 69373 Lyon, France

Address 2: CREATIS (UMR CNRS 5515, Unité INSERM U630) INSA - Bâtiment Blaise Pascal 7, avenue Jean Capelle 69621 Villeurbanne cedex France

\begin{abstract}
Purpose. In this paper, we review several applications of deformable registration algorithms in the field of image-guided radiotherapy.

Materials and methods. We first describe the input and output of deformable registration algorithms. Section 3 is dedicated to a brief review of common methods. Several examples of the use of deformable registration are reviewed in section 4(some equations are given in appendix A). The first four sets of examples deal with intrapatient registration: two sections are dedicated to inter-fraction registration (time interval between images in the order of several days), and the other two sections with intra-fraction registration (displacement during image acquisition or patient treatment, mainly due to respiratory movement; time interval in the order of several tenths of seconds). The last examples (section 4.5) focus on inter-patient registration.

Conclusion. Deformable registration has increasing applications in radiotherapy. Extensive validation of the numerous existing methods is required before extending its clinical use. Nevertheless, deformable registration is already a fundamental image analysis tool for radiotherapy, and will probably be included into all treatment planning systems in the near future.
\end{abstract}

Article published in Z Med Phys soon (2006) 1-38 
Deformable registration is a fundamental image tool that is widely used for the analysis of medical images. Its use in the field of radiation therapy is relatively recent and in constant progression: a search of Pubmed ${ }^{1}$ using the terms "deformable registration" and "radiotherapy" (or related words such as "radiation therapy", "nonrigid registration" ...) has returned about 40 full-length-papers. At ASTRO ${ }^{2}$ and $\mathrm{AAPM}^{3} 2005$ meetings there were respectively 7 and 17 abstracts dedicated to deformable registration in radiotherapy, whereas there had been only 1 and 7 at the 2004 meetings. Usage of deformable registration is mainly found in conjunction with Image-Guided Radiation Therapy (IGRT $[1,2]$ ). The principle of IGRT involves an image-based monitoring of changes in the shape and position of organs during treatment. Images can be acquired using a large variety of modalities: regular CT or in-room-CT (such as CT-on-rails [3] or Cone-Beam CT [4]), MegaVoltage-CT [5], 2D images (either MV or KV), US, PET, MRI, video, etc. The goal of using these images is to reduce treatment margins, allowing to potentially perform safe dose escalation and hopefully improve patient treatment.

This paper is organized as follows. Section 2 describes the input and output of a deformable registration. Section 3 describes various algorithms used to perform deformable registration. Section 4 is dedicated to the use of deformable registration in radiotherapy.

\section{What is deformable registration?}

Image registration is the process of defining a mapping between two images so that the coordinates in one image correspond to those in the other. When the mapping contains deformations, one speaks of deformable registration (DR). A DR takes as input two (or more) images. One image is considered as the reference (or the target image) and the other one is the deformable (or object, or moving, or floating, or test) image. We denote $I$ the reference image and $J$ the deformable image. The output is a transformation $\phi$ which relates the content of the first image to the content of the second image. Let $\mathbf{x}=\left(x_{1}, x_{2}, x_{3}\right)$ (for a $3 \mathrm{D}$ image) be the coordinates of a point in $I, \phi(\mathbf{x})=\mathbf{x}^{\prime}$ is the corresponding point in $J: I(\mathbf{x})=J(\phi(\mathbf{x}))$. DR is related to rigid registration $(\mathrm{RR})$ which is now part of most treatment planning systems. The goal of $\mathrm{RR}$ is also to relate two images by modeling the displacement between the two images. RR often refers to strictly rigid, linear or affine transformations. In the case of rigid transformation, $\phi$ is parameterized with six numbers: three translation and three rotation parameters. A linear transformation is such that $\phi$ can be written as a $3 \times 3$ matrix (9 parameters) $\phi(x)=\mathbf{A x}$. In the case of affine transformation, $\phi$

\footnotetext{
1 http://www.ncbi.nlm.nih.gov/

2 American Society for Therapeutic Radiology and Oncology

3 American Association of Physicists in Medicine
} 
is calculated from 12 parameters: $\phi(x)=\mathbf{A x}+\mathbf{b}\left(b \in \mathbb{R}^{3}\right)^{4}$. By comparison, DR output is a deformation field (DF) of vectors, indicating the correspondence between each voxel in the first image and each corresponding voxel in the second. Such DF can be represented in different ways (see section 3.2), but hundreds or thousands of parameters are generally required. For example, the DR of all voxel displacements in a $512 \times 512 \times 100$ image involves 78 million parameters (three coordinates per voxel). Even if it is not necessary to compute the displacement vector of each individual voxel, $\mathrm{DR}$ is at least one order of magnitude more complex than RR.

A DF is difficult to visualize because of the high number of parameters. In the following, all examples were obtained by DR between a reference end-inhale CT and an end-exhale CT, both acquired in breath-hold. The deformation $\phi$ can be applied to one image in order to simulate the other (warping), such that all corresponding voxels have the same coordinates in both images. The deformed image can be used to perform data fusion by building a composite image displaying information from both images using transparency color; this is typically used for CT/PET images. A DF can also be represented by a warped regular checkerboard (see fig. 1), alternating squared portions of each image. The quality of a DR can be visualized by computing a difference image (see fig. 2) between reference and warped image. It is also possible to represent a DF by displaying vectors on image slices. Hence, only a planar displacement (two of the three dimensions) is represented on each slice. By displaying other slices, with different orientations, it is possible to visualize the 3D deformation (see fig. 3). Warped meshes, see fig. 4, also allow to visualize local deformations. Computing the Jacobian of the DF for each voxel may allow to obtain a grey level representation of deformation amplitude (see fig. 5). The Jacobian is the determinant of the Jacobian matrix (first partial derivatives). It is equal to 1 if the volume does not change locally, it is lower than 1 if there is a local shrinkage and greater than 1 if the volume increases. However, even a Jacobian value of 1 does not mean that there is no displacement, because the Jacobian is invariant with respect to displacement without deformation. More elaborate methods exist for displayin DF, such as Line Integral Convolution [6] or more complex flow visualization techniques $[7,8]$.

$\overline{4}$ The affine case corresponds to a linear case when transformations are described in a homogeneous way, $\mathbf{x}=\left(x_{1}, x_{2}, x_{3}, 1\right)$, by a single $4 \times 4$ matrix. 


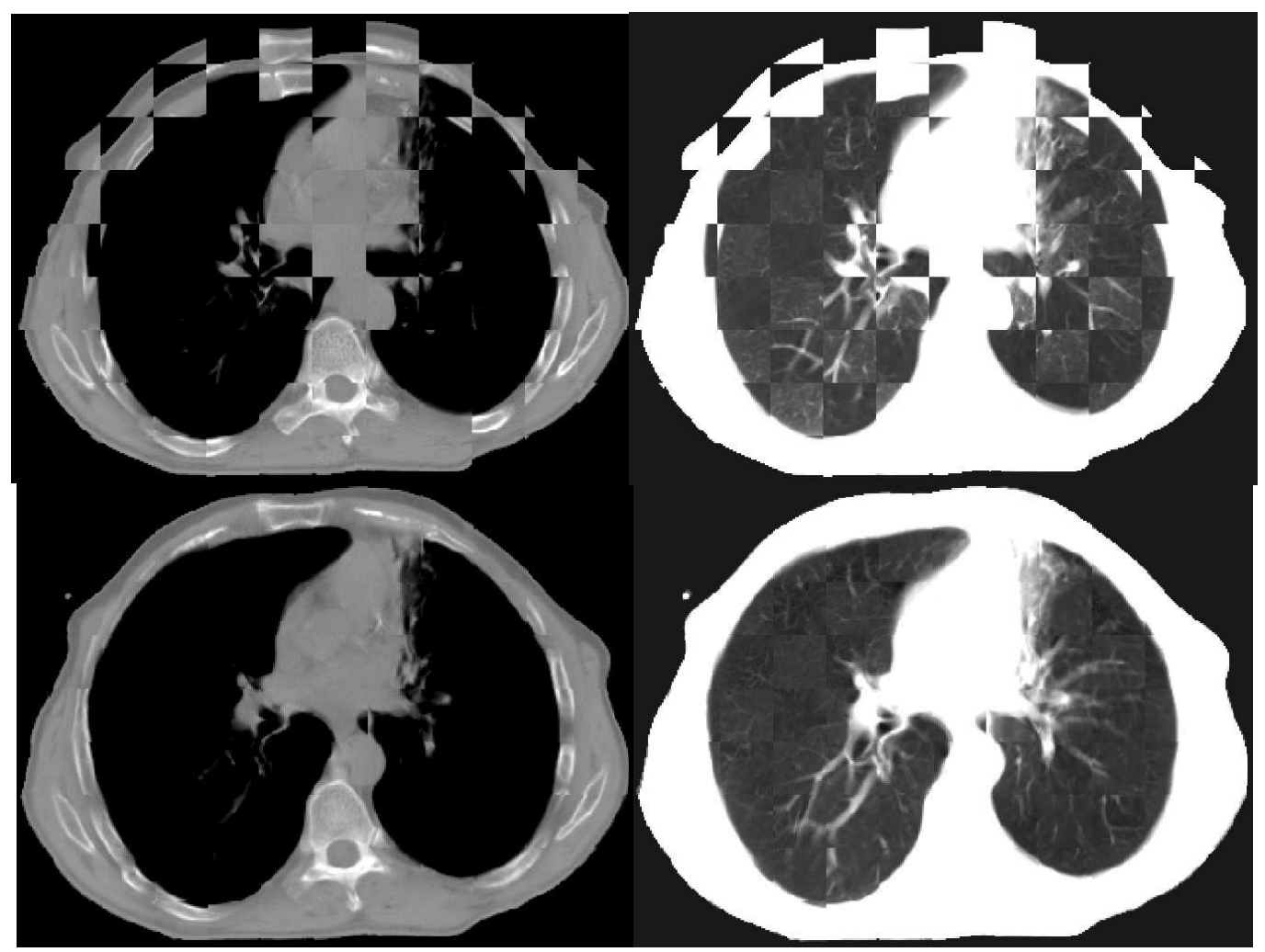

Fig. 1. Checkerboard representation. First line depicts alternative squared portions of $I$ and $J$ (before DR). Second line depicts checkerboard between reference $I$ and warped $J$ according to $\phi$. Left column: images are displayed using soft tissue enhanced grey scale; right column: the same images are displayed using lung-enhanced grey scale.

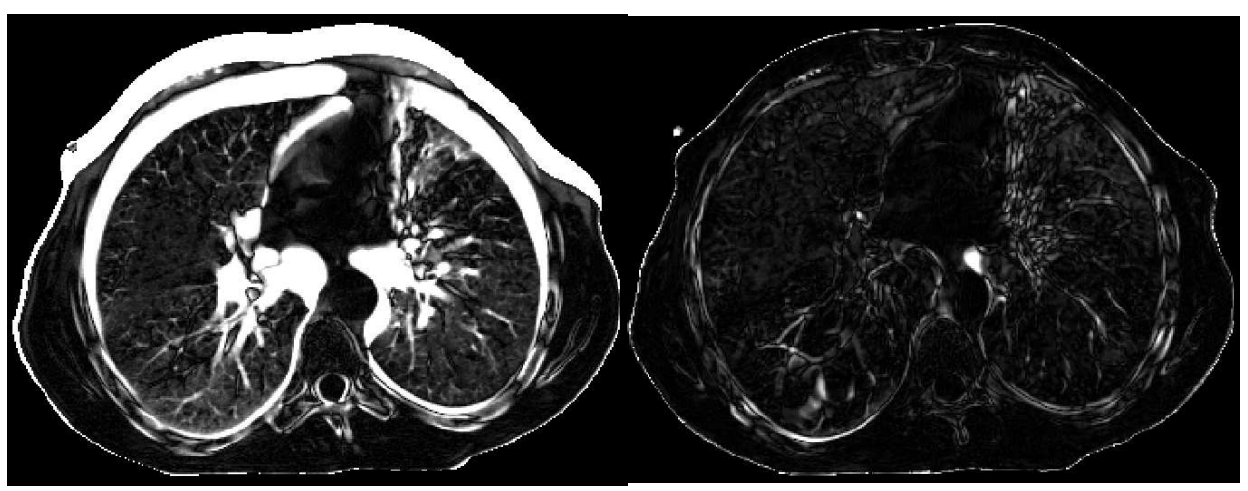

Fig. 2. Differences between images before (left) and after (right) registration. Dark grey levels indicate low image differences, and light grey levels indicate large differences. 


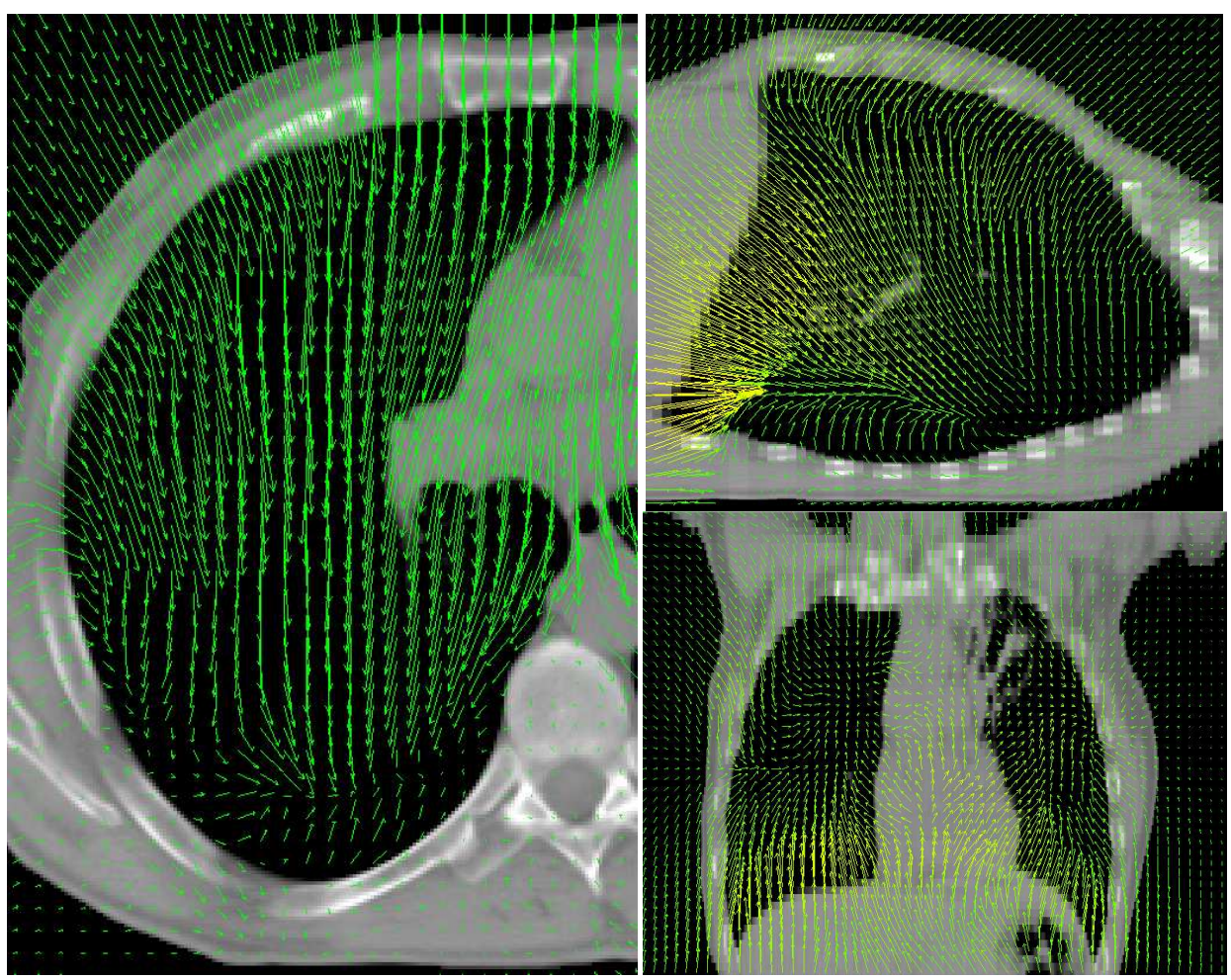

Fig. 3. Vector field representation by superimposition of 2D displacement vectors on several slices. The length of the vectors corresponds to the length of the in-plane displacement. Components of the 3D displacement orthogonal to the slice can be visualized with other slice orientations and with variations of the vector color (from light green to light yellow according to the 3D displacement amplitude). Here vectors are spaced every $7 \mathrm{~mm}$. Sampling of displaying vectors can be changed interactively by the user.
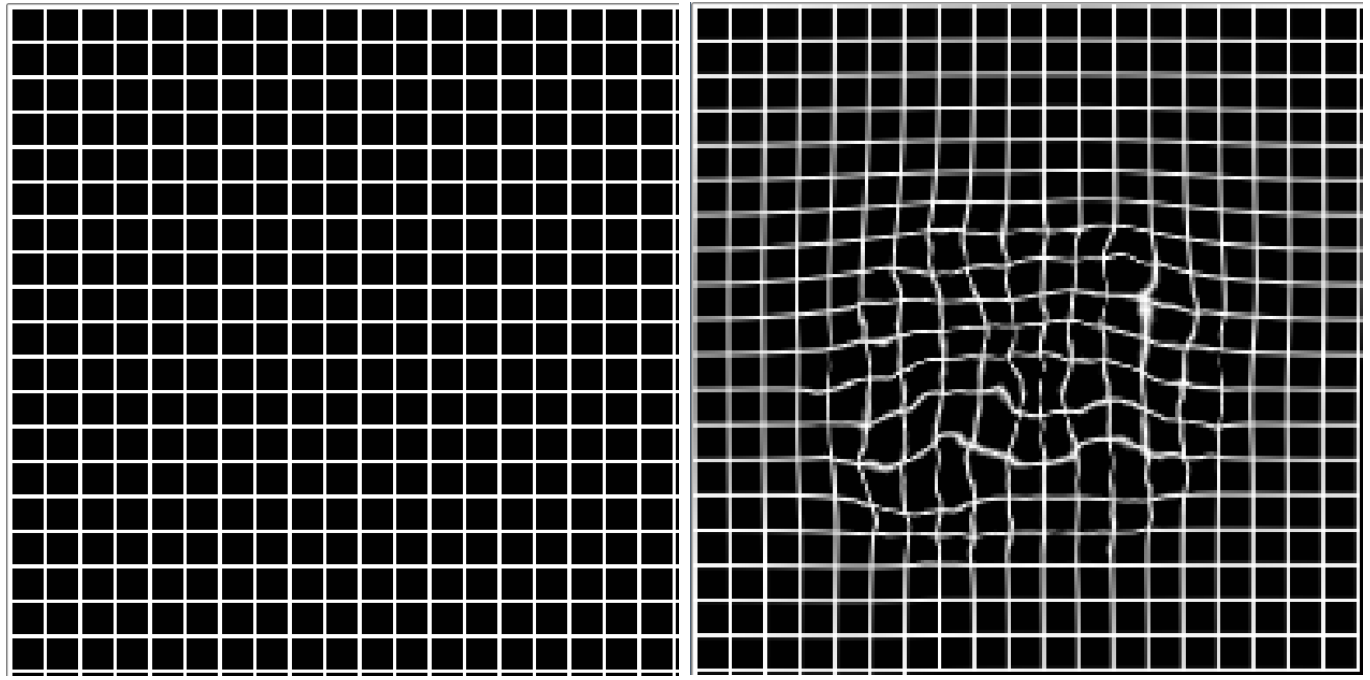

Fig. 4. Regular mesh before (left image) and after (right image) DR. 
Sarrut. 6

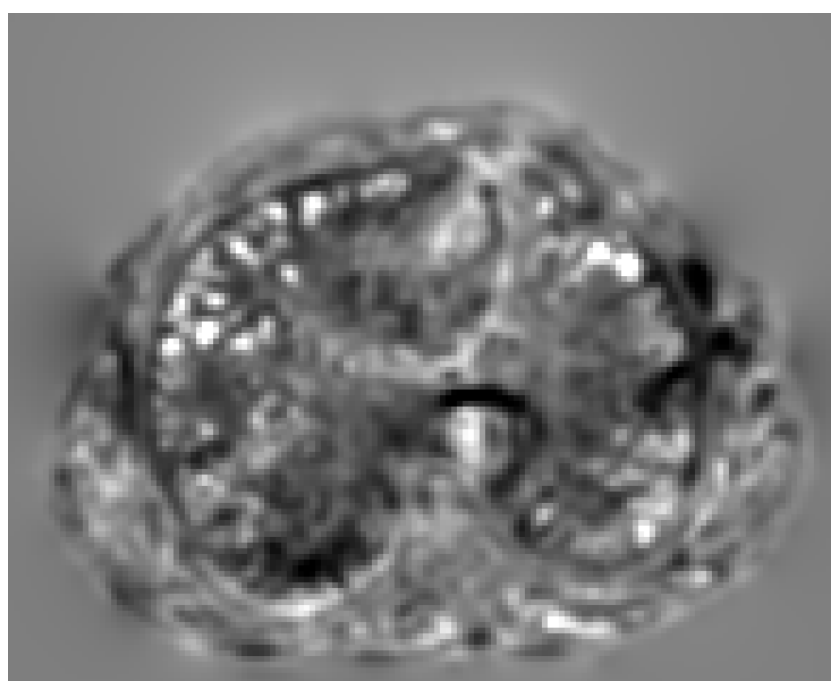

Fig. 5. Grey-level representation of the Jacobian of a DF between from a end-inhale and end-exhale CT images. Values near 1 (no local volume change) are displayed in grey, values lower than 1 (volume decrease) are dark grey to black, and values above 1 (volume increase) are light grey to white. 


\section{How does it work?}

The literature on DR methods is very extensive. The goal of this section is not to provide an exhaustive description of all existing DR methods but to present the main classes of DR in order to understand the contribution of DR methods currently used in radiotherapy. More details can be found in several surveys $[9,10,11,12,13,14,15,16,17,18,19]$. Generally, DR algorithms are described as the combination of several components: a feature space, a similarity measure, a transformation model and an optimization algorithm. The goal is to find an optimal transformation that provides maximum similarity (or minimum distance) between the reference and the deformable image. DR is an ill-posed problem ${ }^{5}$. All methods represent a tradeoff between a certain image similarity (or distance) and an a priori knowledge of the nature (and amplitude) of the deformation to recover.

\subsection{Feature space and similarity measures}

Similarity between images can be feature-based or intensity-based or an hybrid of the two approaches. Feature-based methods use landmark points [20,21], organ contours $[22,23]$ or segmented surfaces. Features must be defined in the two images and must generally be homologous, i.e. each feature in one image is related to its corresponding feature in the other image. Landmarks correspond to identified anatomical points manually selected and paired by an expert or (semi-)automatically extracted [24]. Landmarks may also be selected using image-based processing, typically corresponding to high local gradients. The distance between pairs of features is then defined (as a simple Euclidian distance for pairs of homologous points, or more complex distances for high-order features such as surfaces or lines, including uncertainties or orientations). On the other hand, intensity-based methods involve optical-flow like methods and are generally almost fully automatic. Image similarity is defined as a statistical measure between the intensity (grey-levels) distribution of the two images. For monomodality image registration, other techniques can be used: Sum of Absolute Differences or Sum of Squared Differences (SSD [20,25,26]), or Cross Correlation [27]. For multimodal cases, more advanced measures such as Mutual Information $[28,29,11,30]$ or Correlation Ratio [31,32] can be used but they generally require a longer computation time. Hybrid methods make use of both extracted features and voxel intensities [33] to make the process more robust or to allow user interaction.

5 A problem is well-posed when a solution exists, is unique and depends continuously on the initial data. It is ill-posed when it fails to satisfy at least one of these criteria. 


\subsection{Transformation models}

Sarrut. 8

There are roughly three main groups of methods to model a transformation $\phi$ : global modeling (polynomial, harmonic), semi-local (piecewise polynomial with various splines) and local (regularized dense vector fields) modeling [34] (see table 1). Equations are given in appendix A (page 25). Some authors use global high-order polynomial [35,36], or Harmonic [37] to parameterize the transformation. However, oscillations tend to appear and the global model does not always allow to retrieve local deformation. Many studies have used local piecewise polynomials (splines) as a linear combination of radial basis functions $(\mathrm{RBF})$. The method makes it possible to interpolate or approximate pairs of homologous features defined in each image. Thin-plate splines (TPS) or second order Laplacian splines are the most popular basis functions [38], but other functions such as multiquadrics, inverse multiquadrics or Gaussians can also be used [39]. The TPS coefficients are found by resolving a set of linear equations, which requires the inversion of a matrix which size depend on the number of points to be interpolated or approximated. Other basis functions such as Fourier series [40], wavelets, membrane spline [41], elastic body spline [42], div-curl spline [43] have also been used. Transformation models supported by this approach are mostly interpolant (exact landmarks matching) but an extension to the approximation case has been proposed [44] to take into account landmarks localization uncertainties. Such functions do not have compact support: each landmark pair impacts the transformation result globally. Fornefett et al. [39] proposed the use of compactly supported RBF with Wendland functions. There are many other ways to represent the transformation with local piecewise polynomial models. One of these is B-splines [30,34]. B-splines are piecewise polynomials of degree $n$ (tri-cubic splines are often used, $n=3$ ), with interesting mathematical properties: compact support, continuous $(n-1)^{t h}$ derivative, etc. They are often described by a free-form deformation (FFD) model: deformation is carried out by an underlying uniform regular mesh of control points.

Other widely used approaches consist in representing the transformation with dense vector fields (up to one vector per voxel) and adding regularization constraints to the field. Generally, constraints are defined using an energy function computed from the deformation field. At each iteration of the optimization procedure, the energy of the current transformation is used to impair non smooth transformation. Linear elastic energy is commonly used $[45,40]$. It is based on the physical equations of the deforming material, assuming that the relationship between strain and stress is linear. Other regularizing energies are the membrane or Laplacian model (which can be considered as a simplification of the linear elastic model [46]), bi-harmonic [38] (TPS correspond to an exact solution to this energy minimization), viscous fluid $[47,48,49]$ (same equations as for the elastic model but applied to the velocity field instead of the displacement field), Jacobian-based [50,51], Gaussian [52,48] (which can be related to elastic regularization under some assumptions), etc. Interested readers should refer to Cachier et al. [53] who report that almost all regularization energies are based on the same small set of differential quadratic forms.

Biomechanical models $[54,55]$ do not explicitly use similarity measures. Instead they 
simulate organ deformation by using both physical material properties and constraints based on the initial and final states of the organs. They are based on the Finite-Element Method (FEM) and use similar equations (elastic model for example) to simulate individual organ deformation. Deformation is generally represented by triangular meshes for surface-based models or tetrahedral meshes for volumebased models. The individual material properties of each organ must be described, with parameters such as Young's modulus and Poisson's ratio. Chi et al. [56] studied the relationships between those parameters and achievable registration. Contact between organs is also sometimes simulated [57].

\begin{tabular}{|c|c|c|c|}
\hline \multirow{4}{*}{\multicolumn{2}{|c|}{ Features space and similarity measures }} & \multicolumn{2}{|r|}{ Transformation models } \\
\hline & & Global & $\begin{array}{l}\text { - Affine (almost rigid) } \\
\text { - Polynomial } \\
\text { - Harmonic }\end{array}$ \\
\hline & & $\begin{array}{l}\text { Semi-global (RBF, } \\
\text { piecewise polynomial) }\end{array}$ & $\begin{array}{l}\text { - Non-compact support : TPS, Fourier } \\
\text { series, wavelets, elastic, div-curl } \\
\text { - Compact support : Wendland, B-spline }\end{array}$ \\
\hline & & \multirow[t]{2}{*}{$\begin{array}{l}\text { Local (regularized } \\
\text { dense vector field) }\end{array}$} & \multirow{4}{*}{$\begin{array}{l}\text { - Membrane model (Laplacian) } \\
\text { - Bi-harmonic model (2 }{ }^{\text {nd }} \text { order Laplacian, TPS) } \\
\text { - Linear elastic, fluid } \\
\text { - Gaussian } \\
\text { - Jacobian-based ... } \\
\text { - FEM methods with triangular (surface) or } \\
\text { tetrahedral (volumes) meshes } \\
\text { - Individual organ properties (Young's modulus } \\
\text { and Poisson's ratio) } \\
\text { - Contact-impact analysis }\end{array}$} \\
\hline Features-based & $\begin{array}{l}\text { - Types : points, lines, surface ... } \\
\text { - Selection : manual or automatic } \\
\text { - Pairing : manual or automatic }\end{array}$ & & \\
\hline Intensity-based & $\begin{array}{l}\text { - Local : SAD, SSD } \\
\text { - Neighbourhood : CC, MI, CR ... }\end{array}$ & \multirow[t]{2}{*}{ Biomechanical model } & \\
\hline Hybrid & - Mix IB and FB methods & & \\
\hline
\end{tabular}

Table 1

Short classification of some feature-spaces and transformation models used in deformable registration.

\subsection{Conclusion}

In feature-based methods, the intervention of experts did allow to obtain a confident correspondence between sets of points (even when taking into account inter-observer variability), but this method remains time consuming, error prone and not appropriate for a daily use. The difficulties were to establish a correspondence between landmarks and to insure that extracted landmarks in the two images correspond to same physical points. Moreover, landmarks should be uniformly distributed within the volume in order to correctly infer the deformation. However, such methods are generally faster than intensity-based methods. Intensity-based methods do not require (manual) segmentation of organs but are subject to image artifacts. Biomechanical models require the definition of organ properties which are generally not well known and are thus to be found heuristically.

There is no single method outperforming all other methods and which can be used in all situations. The result is always a tradeoff between accuracy and speed. The application can help choose a DR as a function of a priori differences in images. The differences between images that we want to be registered can be due to several factors: change in patient anatomy (intra-subject registration), different image viewpoints, images from different sensors (multimodality registration), images from 
different patients (inter-subject registration), dynamic motion (intra-fraction motion such as breathing). The type and amplitude of transformation $\phi$ depends on such a priori differences. A priori information on the nature of the deformation is therefore crucial. The next section describes several applications of DR.

\section{What use in radiotherapy ?}

DF is used in a great variety of processes in IGRT. This section does not provide an exhaustively list of DR applications in radiotherapy but describes a number of examples. The first example describes local organ deformations induced by the application of an endo-rectal coil. The second example deals with inter-fraction organ deformations. The third example describes intra-fraction motion mainly due patient respiration. Other examples include multi-modal DR (PET/CT) and interpatient segmentation propagation with DR.

\subsection{Example $n^{\circ}$ 1: fusion of functional MRSI to anatomical CT or MRI for prostate treatment}

DR is used to combine anatomical data obtained by MRI or CT and functional information from magnetic resonance spectroscopic imaging (MRSI) for prostate treatment planning. MRSI provides information on the in vivo metabolic activity of tissues that can be related to the presence of cancer [58]. Prostate cancer cells lack the ability to produce and accumulate citrate, and MRSI is used to visualize regions with no or low citrate concentration. In practice, however, the use of endorectal probes for MRSI distorts the prostate and other neighboring soft tissue organs, modifying their shape when compared to an anatomical MRI or a planning CT (see fig. 6). It makes the analysis and the use of MRSI in treatment planning difficult and RR insufficient. DR is thus an adequate tool to recover the deformation, as it allows a combination of functional and anatomical information.

Lian et al. [59] used TPS to interpolate the deformation from 4 to 8 homologous control points in each pair of slices. DR is only performed slice by slice in $2 \mathrm{D}$, potentially resulting in errors in the longitudinal direction. Control points were chosen manually along the contour of the prostate gland and feature points such as corners and intersections of edges were identified. Based on a similar approach, Venugopal et al. [58] reported a registration method that required contouring of the gross tumor volume (GTV) in both inflated and deflated images. They also used 2D TPS from a manual and contour guided selection of homologous control points. Wu et al. [36] used an intensity-based DR between MRSI and MRI images which did not require contouring the organs. They used mutual information as similarity measure and the deformation was parameterized by a global polynomial function of order 5-6 (168-252 parameters). The regularization energy was the bi-harmonic differential operator, an energy associated with the TPS, involving second partial 

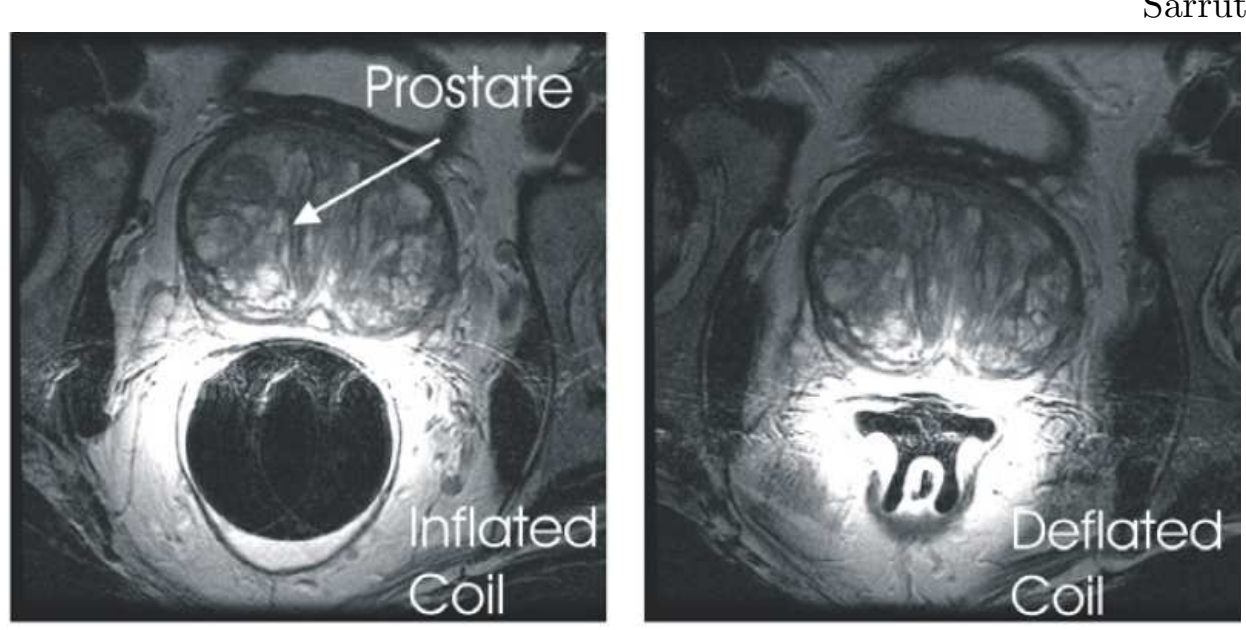

Fig. 6. In the left MRS image the endorectal coil is inflated with $100 \mathrm{~cm}^{3}$ of air, while in the right image the endorectal coil is completely deflated. From Venugopal et al. [58].

derivatives. For MRSI to CT image registration, Schreibmann et al. [60] proposed an hybrid method combining contour-based free form deformation using B-spline and information on pixels contained in narrow bands around the contours. This method allowed to avoid the use of homologous control points. Intensity-based information was done with normalized correlation as similarity measure and optimization was performed with a quasi-Newton approach allowing to avoid the computation of the Hessian by using an iteratively updated approximated matrix [61].

In each approach, $\mathrm{RR}$ was considered inefficient to retrieve deformation. Hence, DF was used to warp back MRSI data to anatomical data obtained from MRI or CT by removing the deformation, thus allowing image fusion(see fig. 7).

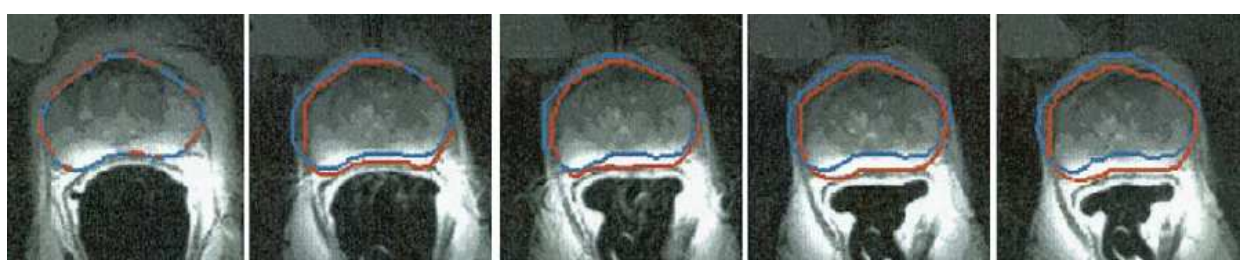

Fig. 7. Image of the prostate using the endorectal coil with varying degrees of inflation (40,30,20, 10, $0 \mathrm{cc}$, respectively). A distortion of the prostate is visible. The blue contour obtained at maximum inflation is used as a reference. The red contour delineates the prostate area at different levels of inflation. The blue contour is overlaid on all images to show the amount of distortion. From Wu et al. [36].

\subsection{Example n०2: IGART}

Fraction to fraction variations of patient anatomy and setup lead to dosimetric uncertainties, potentially leading to under-dosage of the tumor and/or over-dosage of healthy tissues. It might be even more problematic for intensity-modulated radiation therapy because of higher gradients and potentially higher doses. The anatomy 
of a patient can evolve during the course of the treatment for several reasons: tumor shrinkage or growth, patient weight loss, variations of internal soft organ configuration such as caused by the filling of the bladder, etc. Due to this organ motion and several other reasons, the delivered dose might not be identical to the predicted dose. Adaptive Radiation Therapy (ART) $[62,2,63]$ was developed to reduce these uncertainties using information obtained frequently during the treatment course and making mid-course adjustments. Reducing uncertainties would allow to reduce margins, allowing to potentially perform safe dose escalation and hopefully improve the treatment. DR can be used to (semi-)automatically quantify image to image variations and potential dosimetric gains and thus represents a key tool in IGART.

Treatment planning is based on an anatomical representation of a patient generally obtained from a CT image acquired before treatment. Following the notations proposed in [64], let $\mathbf{A}\left(t_{0}\right)$ denote the anatomical representation of one patient at time $t=t_{0}$. Several studies $[2,64,65,66,54,57]$ (among others) aimed to adapt the initial treatment plan to accommodate changes in patient anatomical configuration revealed by a post-treatment follow-up image, $\mathbf{A}\left(t_{1}\right)$, acquired for example after one week. To be able to potentially check, adapt, correct or modify the treatment according to the current situation, one needs to compute an estimation of the dose deposit $d_{1}$ in $\mathbf{A}\left(t_{1}\right)$ and compare with the previous dose map $d_{0}$ corresponding to $\mathbf{A}\left(t_{0}\right)$ in order to obtain a difference dose map $\Delta d_{01}$. There are two issues when performing this first step of an ART strategy. First, new shapes and positions of organs and treatment targets must be estimated in order to estimate the current dose map $d_{1}$. Secondly, a cumulative dose map must be computed, which cannot be done by simply subtracting the dose value of each voxel $\left(\Delta d_{01}(\mathbf{x}) \neq d_{0}(\mathbf{x})-d_{1}(\mathbf{x})\right)$. For both issues, DR can provide useful information.

\subsubsection{Estimation of anatomical variation using $D R$}

One way of estimating the current dose $d_{1}$ is to re-compute the dose deposit as a function of initially planned irradiation parameters but using the new anatomical configuration $\mathbf{A}\left(t_{1}\right)$. Hence, organs and target contours required to perform dose computation need to be redefined on $\mathbf{A}\left(t_{1}\right)$. It is possible to import the contours drawn on $\mathbf{A}\left(t_{0}\right)$ into $\mathbf{A}\left(t_{1}\right)$ and to use them as initial input for additional manual recontouring. Instead, DR can be performed between $\mathbf{A}\left(t_{0}\right)$ and $\mathbf{A}\left(t_{1}\right)$, and the resulting DF is used to propagate contours. The result of this step is a set of contours in $\mathbf{A}\left(t_{1}\right)$ corresponding to those drawn in image $\mathbf{A}\left(t_{0}\right)$. Such contours can still be checked and corrected manually but they are potentially closer to the solution than simple imported contours. The dose distribution $d_{1}$ could then be computed according to the initial irradiation parameters.

For prostate treatments, several DR methods have been proposed to perform this first step. Yan et al. [22] proposed a method to estimate organ deformation using a biomechanical model. The contours of each organ of interest were manually drawn (then smoothed with a cubic-spline) and a set of fiducial points was manually selected. A 3D mesh (with tetrahedronal volume elements) was then generated and deformation was obtained by FEM solution of the differential equations of the 
biomechanical model including mechanical properties of the tissue. The method was used by Birkner et al. [54] on CT images of patients with large rectum and bladder motion. Schaly et al. [23] also used a contour-based dose mapping technique, but theirs was based on TPS. An automated heuristic technique was proposed to generate homologous control points on contours, including tumor contour, all surrounding critical structures and external contours. For each contour, 3D deformation was propagated to the whole volume by TPS interpolation. This approach was validated in prostate patients [67]. Fei et al [68] proposed an almost fully automated DR of prostate and pelvic MR volumes for image-guided prostate cancer treatment. They used a semi-automatic method to detect corresponding pairs of feature points, beginning with a manual localization of some points (prostate center, two hip joints, two distal femurs) then automatically creating several hundreds of other feature points. Local rigid transformations were optimized using the mutual information similarity measure computed on each small cubic area centered on points. Final deformable transformation was obtained by TPS interpolation of corresponding control points. Wang et al [69] proposed an intensity-based DR to register prostate motion on a daily basis in order to aid dose tracking(see fig. 8). The method used an optical flow-like algorithm inspired from [52] in order to register two CT scans. A reverse similarity force taking into account both image gradients was added to speed up the calculation. Validation of this approach was studied in [70].

It should be noted that several studies have demonstrated that the deformation of prostate and seminal vesicles during the course of radiotherapy is small relative to organ motion [71,72](see fig. 9). Hence, DR is not strictly required and RR can be sufficient when performed locally, relatively to other structures. This can be done using an automated intensity-based RR method, as proposed in [73]. Most of the studies presented here were focused on the pelvic region, but other localizations could potentially benefit from the same type of techniques: breast [74,75] (see fig. 10) or lung [76,77] (see section 4.3).

\subsubsection{Dose accumulation computation using deformation field}

The previous step was dedicated to obtaining quantitative information on organ/target deformation between two anatomical representations. In order to compare two dose distributions $d_{0}$ and $d_{1}$, respectively estimated according to two anatomical representations $\mathbf{A}\left(t_{0}\right)$ and $\mathbf{A}\left(t_{1}\right)$, a difference dose distribution $\Delta d_{01}$ should be computed by expressing the dose in the same spatial reference, $\mathbf{A}\left(t_{0}\right)$ for instance. By using the DF $\phi$ obtained from a DR, the difference dose distribution can be expressed as $\Delta d_{01}(\mathbf{x})=d_{0}(\mathbf{x})-d_{1}(\phi(\mathbf{x}))$. Hence, by integrating several patient descriptions $\mathbf{A}\left(t_{i}\right)$ over the course of the treatment, several authors proposed formulations of the planning optimization problem which included dynamic geometrical variations of the organs. Following this principle, Yan et al. [22,78] were among the first to propose a model for accumulating fractionated dose in a deforming organ. A similar dose warping technique was proposed by Schaly et al. [79], or Wu et al $[64,65,66]$, using the DR method published by Lu et al. [25]. Mohan et al. [80] used a similar method but exploited it to register synthetic Beam's-Eye-View (BEV) 2D images (see fig. 11). These images were computed for each beam, segmented into 
Sarrut. 14

(a)

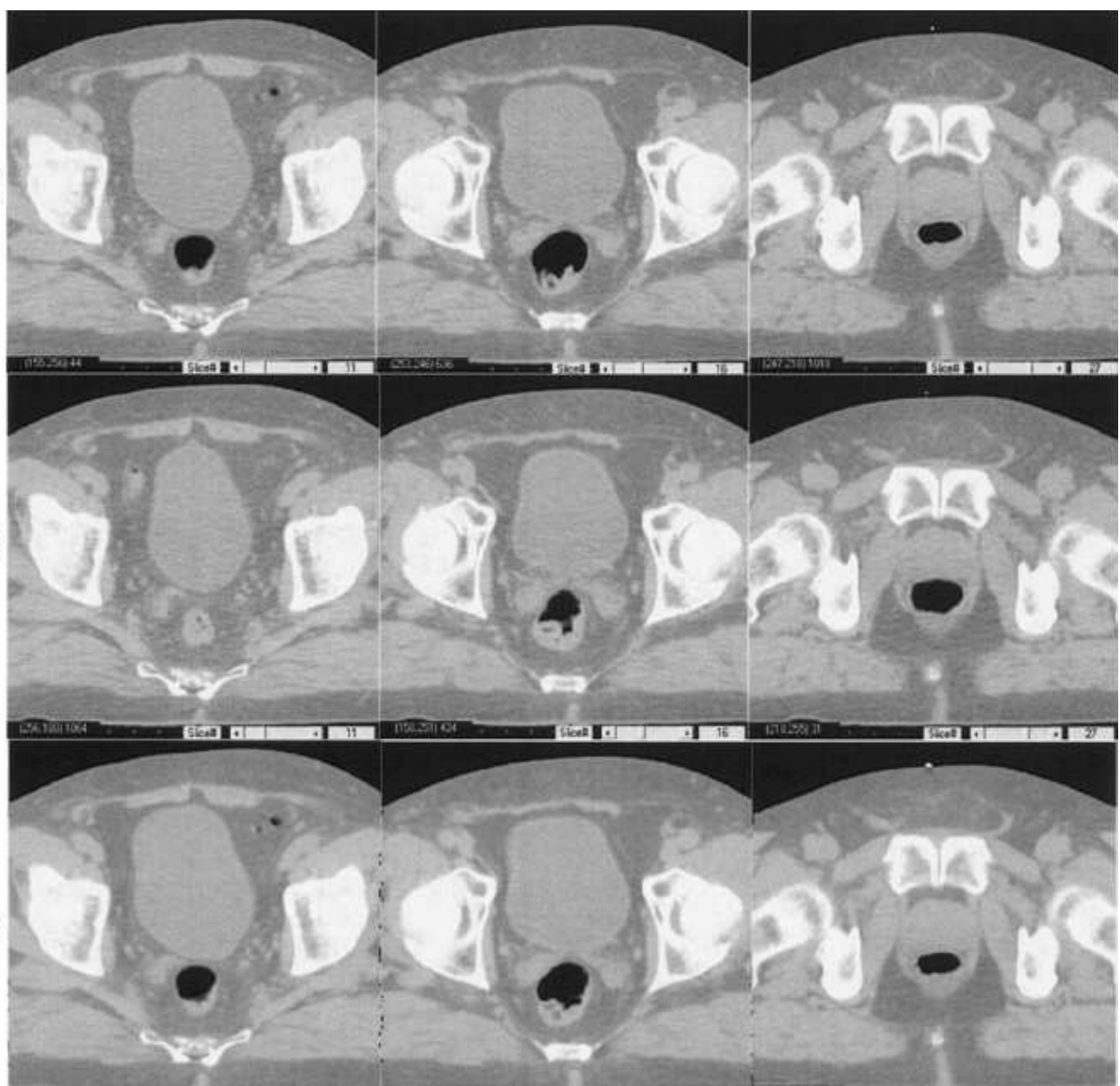

Fig. 8. DR of images obtained from two separate CT sessions. (a) three slices of pelvic CT images from the first CT session. (b) Corresponding slices from the second $\mathrm{CT}$ session. (c) Images from the second CT warped according to the resulting DF to match images from the first CT. From [69]

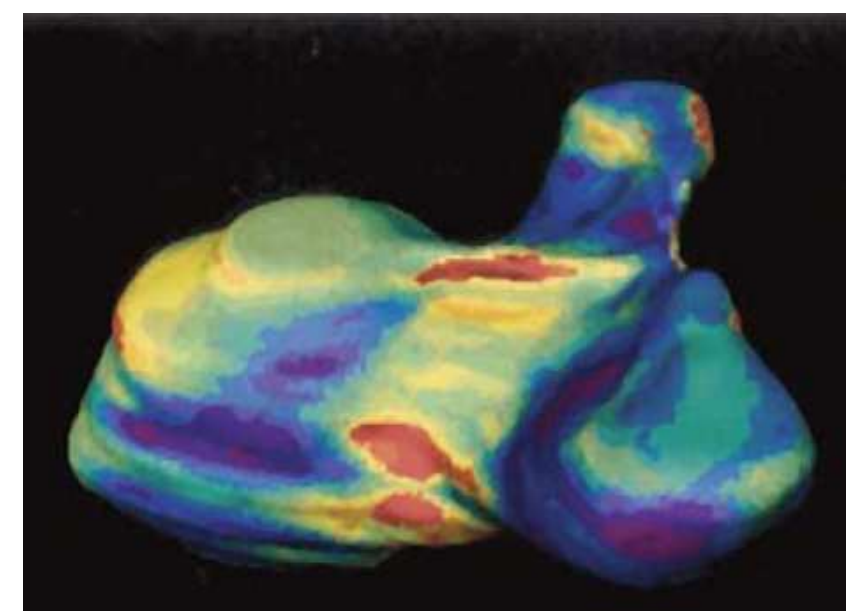

Fig. 9. Measured local shape variation displayed as a color wash on patient average gross tumor volume (GTV) From [71] 
Sarrut. 15
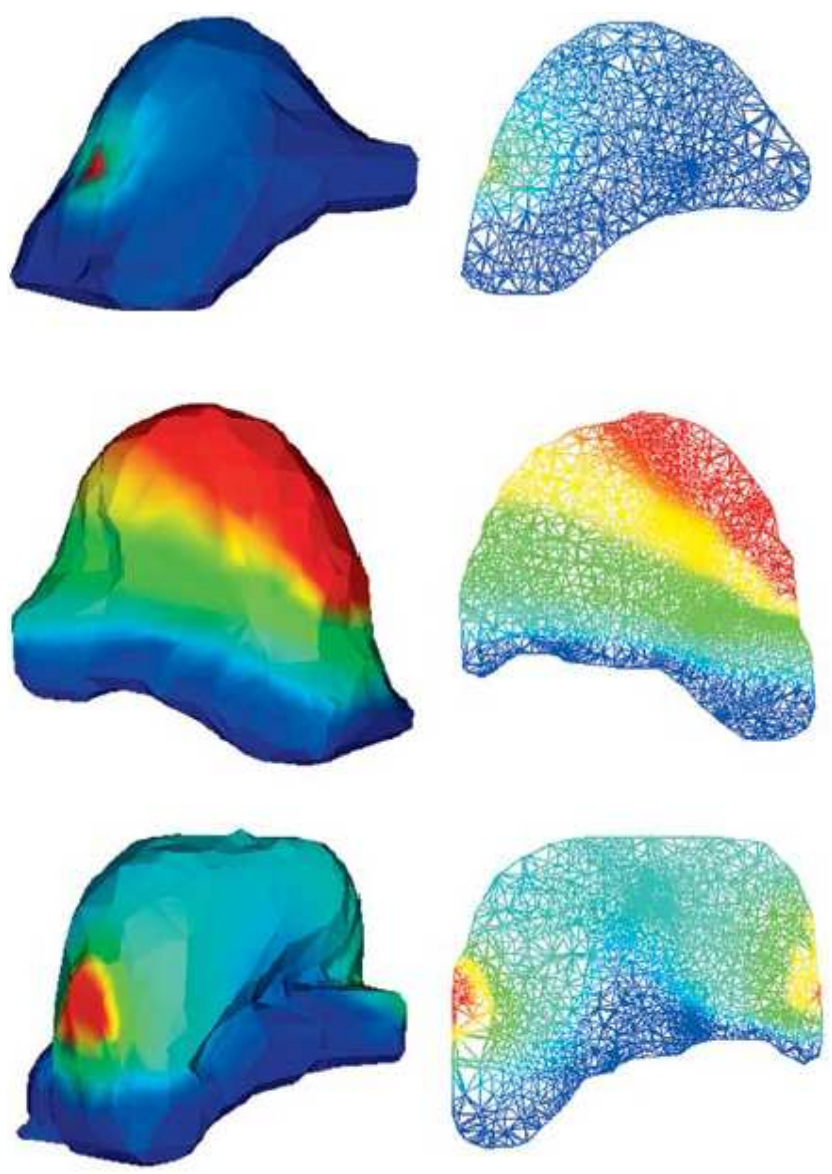

Fig. 10. Surface and wire-frame renderings of example solutions for FEM models of breasts. (from top to bottom) Patient cases 1-3, for point puncture (case 1), regional displacement (case 2), and two-sided contact (case 3). (left) Surface renderings of deformed meshes. (right) Wire-frame cuts through deformed meshes. Deformation magnitudes is mapped using rainbow color coding, with dark blue corresponding to $0 \mathrm{~mm}$, and dark red corresponding to 10-mm deformation. From Schnabel et al. [74]

regions of the planning target volume (PTV) overlapping normal critical structures. Intensity distributions within each segment from the original pretreatment intensity-modulated radiotherapy plan were mapped to the corresponding segment within the treatment BEV. The grey-scale images were then used as input of the DR algorithm in order to deform intensity distribution by using TPS interpolation inside the segment region.

\subsubsection{Conclusion}

Taking into account organ/target inter-fraction motion could help improve the treatment [2]. Dosimetric consequences of including fraction to fraction organ motion and deformations into the treatment is a different matter and has been evaluated in several studies $[81,82,83,79,84]$. The main ideas are that accounting for daily change in the anatomy would allow to escalate the prescription dose, but the dosimetric impact is patient-dependent. Such ART processes require to extract quantitative 


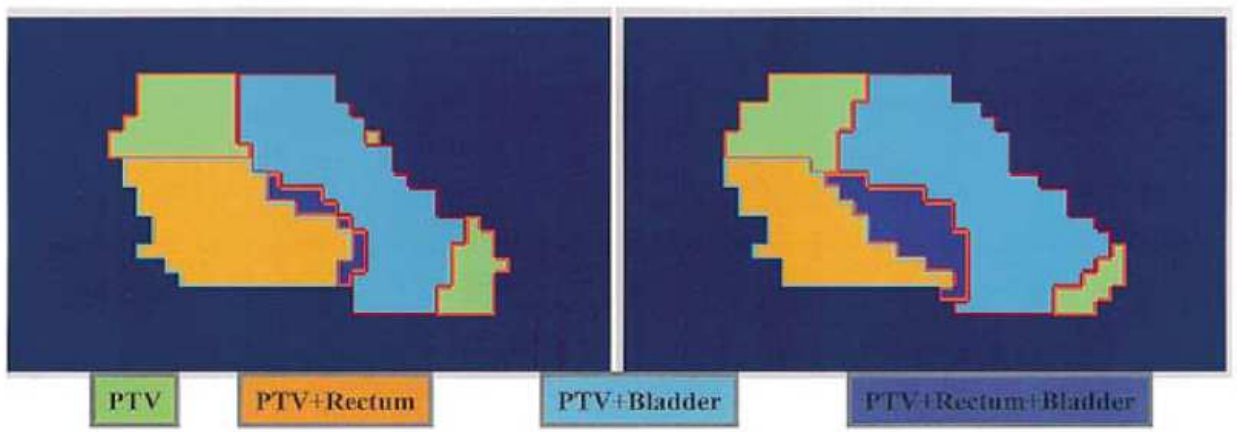

(c) Initial Intensity Distributions

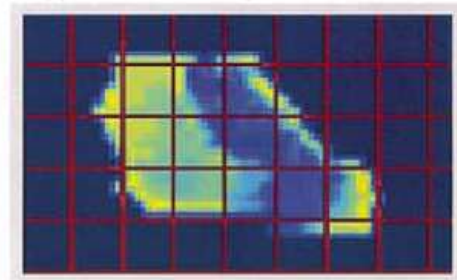

(d) Deformably Mapped to Fraction 7

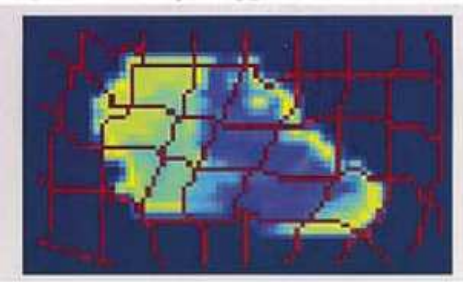

Fig. 11. Illustration of aperture-segment-by-aperture-segment deformation of intensity distributions. The beam's-eye-view (BEV) apertures for each beam are segmented into regions of the planning target volume (PTV) overlapping normal critical structures. Intensity distributions within each segment from the original pretreatment intensity-modulated radiotherapy plan are mapped to the corresponding segment within the treatment BEV. From [80]

information from several images using registration methods. It is worth noting that rigid motion (patient setup error) is probably the first cause of uncertainties and leads to the most dramatic dosimetric consequences. Nevertheless, DR remains required to accurately determine dose accumulation in the organs (such as in the rectum or the bladder, see fig. 12). Kupelian et al. [72] also reported infrequent but significant deformations. Finally, the rigid displacements of soft tissues are local and can generally not be retrieved using global RR. Global intensity-based DR methods such as in [69] or biomechanical models such as in [22] potentially remain an alternative to take into account both issues (rigid and deformable motion) at the same time.

\subsection{Example n' 3: temporal breathing deformation (liver and lung)}

Accounting for intra-fraction organ motion is an important challenge in lung cancer radiation treatment [85]. Movement and deformation due to breathing cause problems with image acquisition (distorted target volume [86]), treatment planning and radiation delivery [87]. Motion potentially leads to GTV underdosage and/or unnecessary irradiation of healthy tissues. As with ART, reducing uncertainties in target position should result in a decrease in healthy lung irradiation and should allow tumor dose escalation, which could potentially lead to better outcome [88]. Motion management [63] includes several steps [89,87]: motion limitation (contention, 


\section{$\mathrm{AP}(\mathrm{cm})$}

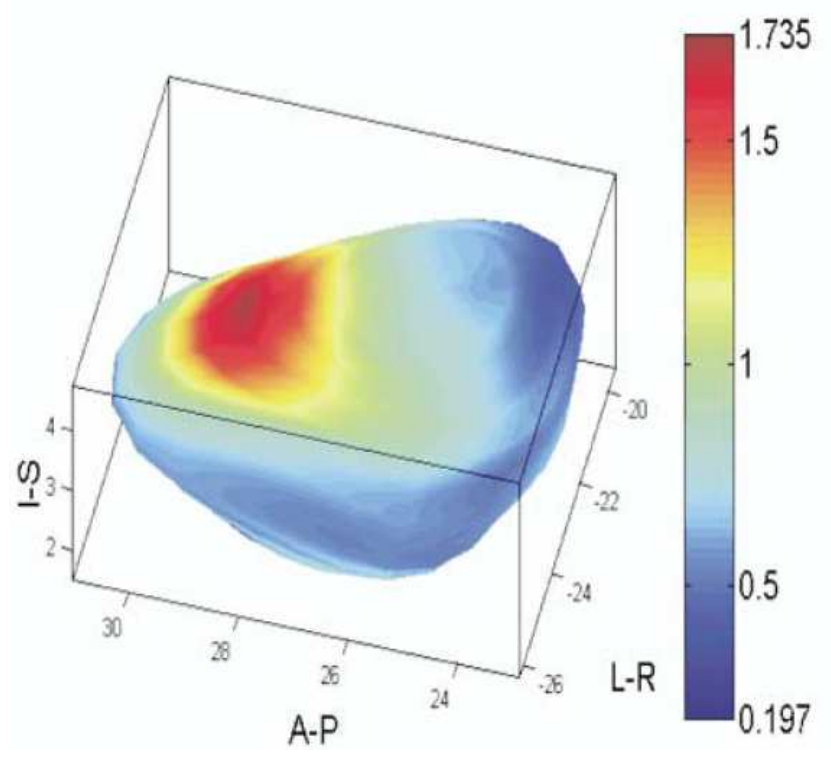

Fig. 12. Bladder displacement distribution measured by using the standard deviation $(\mathrm{cm})$ in the anteroposterior direction. From [2]

breath-holding, coached breathing, gated radiation delivery), residual motion quantification (intra and inter-fraction), residual motion management (adapted dosimetry) and treatment delivery control (QA). Dealing with breathing motion in an IGRT context involves several image modalities such as: blurred, slowly acquired CT image averaging breathing periods, end-inhale/end-exhale/deep-inspiration (EEXEIN-DI) breath-hold CT images, 4D CT images, 2D+t dynamic projective images (mega-volt portal images or kilo-volt radiographic images acquired with on-board cone-beam, for instance), etc.

Recovering motion from images representing thorax anatomy at several breathing states is a process similar to recovering motion from day to day images (previous section). However, several properties of the motion make the process somewhat different. There is a temporal coherence of the motion between different states. Let $\mathbf{A}\left(t_{i}\right), \mathbf{A}\left(t_{j}\right), \mathbf{A}\left(t_{k}\right)$ be three successive states. In the previous section, motion $\phi_{i j}$ between anatomical representations $\mathbf{A}\left(t_{i}\right)$ and $\mathbf{A}\left(t_{j}\right)$, and motion $\phi_{j k}$ between $\mathbf{A}\left(t_{j}\right)$ and $\mathbf{A}\left(t_{k}\right)$ were independent $\left(t_{j}-t_{i}=\right.$ days). In the case of breathing, $\phi_{j k}$ should be coherent with $\phi_{i j}$ because the two motions occur at short time interval $\left(t_{j}-t_{i}=\right.$ few tenths of second). Motion from breathing also happens with some degree of regularity and changes in lung tissue density (due to the inspired air) occur on a fairly regular basis. This section deals with the use of DR to account intra-fraction breathing motion of the liver and lung. 


\subsubsection{Breathing deformation of the liver}

Kitamura et al. [89] showed that liver tumor motion can reach $10 \mathrm{~mm}$ in tidal breathing. Yan et al. [81,22] showed that linear (rigid) registration was insufficient to recover the liver deformation caused by diaphragm motion. Instead, they applied their previously described biomechanical model to the liver. They determined the liver boundary points in relation to the surrounding anatomic structures. Brock et al. [90] proposed a linear elastic, small deformation, mechanical system to construct a $4 \mathrm{D}$ model of the liver during breathing. The finite-element model was build from two EEX-EIN breath-hold CT images (see fig. 13)with a volumetric mesh (6000 tetrahedral finite elements) obtained from initial contours using biomechanical properties (see fig. 14). Liver was split into six regions (segmentation defined from a standard anatomic reference system) and different initial constraints were applied to each region. Brock et al. used an equivalent model with six intermediate positions (from $t_{0}$ to $t_{5}$ ) to study dose accumulation in the liver deformed by breathing [91]. The model was extended in [92] into a finite element model-based DR method named MORFEUS (with MRI). This multi-organ model (liver, spleen, external surface, stomach and kidneys), describes the surface interface between organs and assigns each individual organ material properties that allow accurate deformation of internal structures. The same authors [29] proposed another, less time-consuming and user-dependent method, based on TPS interpolation of control points after initial rigid alignment using mutual information. The control points were automatically placed using a probabilistic atlas (created from 32 patients). Rohlfing et al. [93] also proposed a model of liver motion during respiratory cycle with an intensity-based approach. They used a 4D dataset, obtained with gated MRI, from EEX to EIN, with 8 instants. Motion was modeled using an intensity-based DR method similar to the one presented in [30] (on breast). Deformation was parameterized with a free-form deformation of cubic B-spline, and computed between the reference EEX image and each successive 3D image of the dataset.

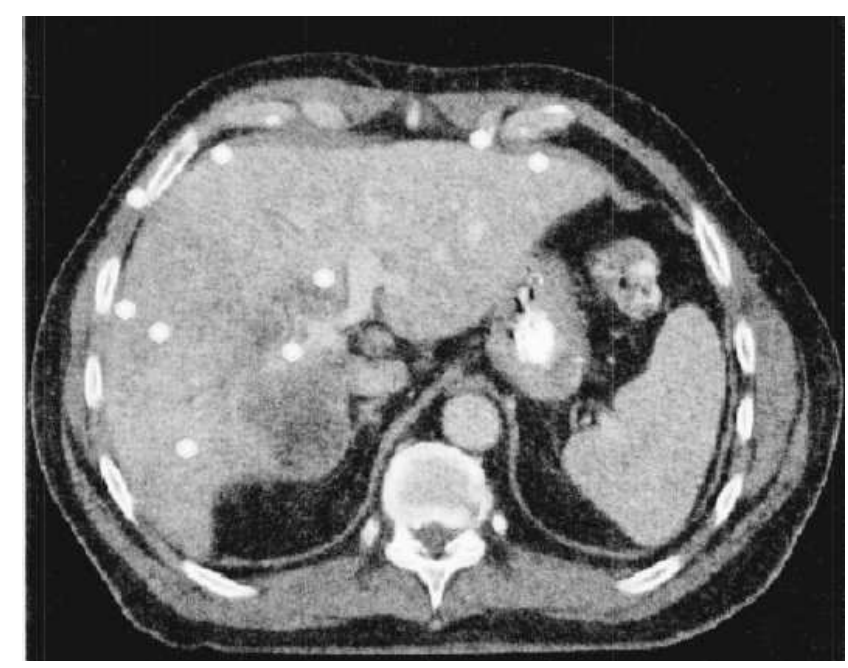

Fig. 13. CT slice of the liver. From [29] 
Sarrut. 19

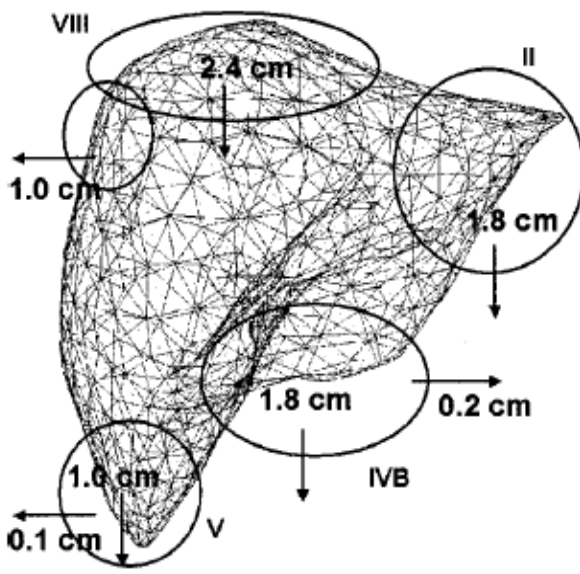

(a)

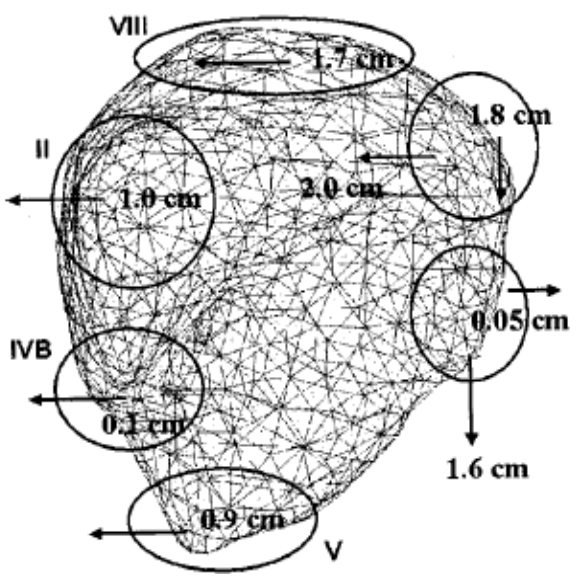

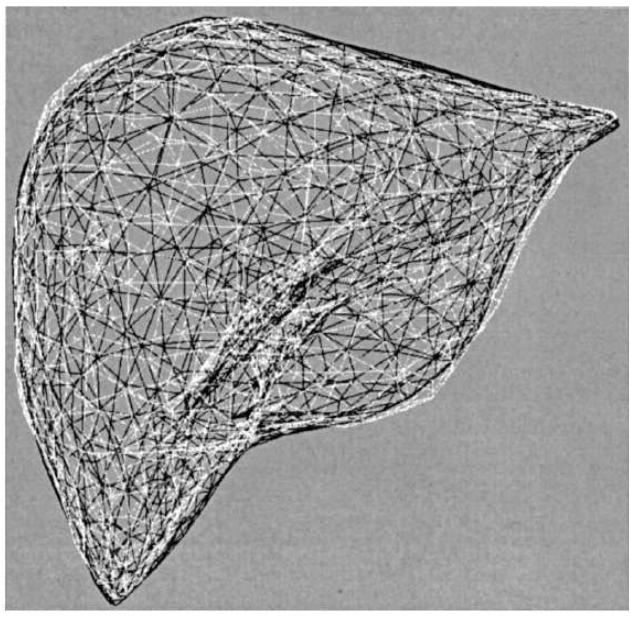

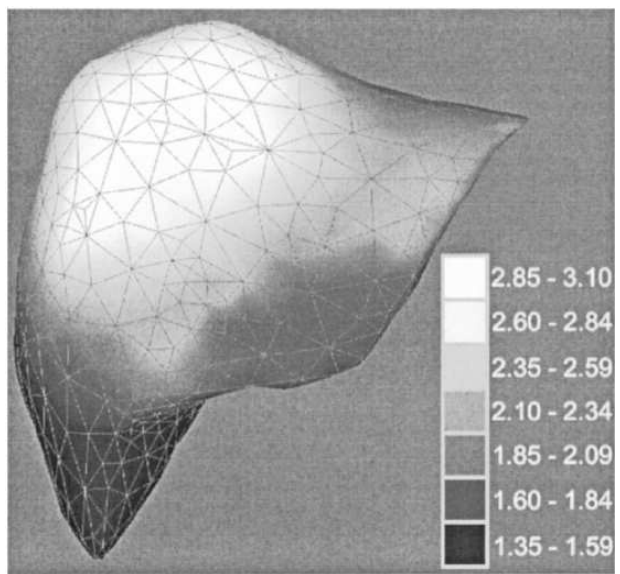

Fig. 14. Left column images show anterior-posterior (top) and lateral (bottom) views of the liver at end-exhale with regions and displacements. Top-right image shows modeled inhale position (in white), and actual patient liver at inhale (in black). Right-bottom image shows displacement map of end exhale liver to modeled end inhale position. Displacements are vector magnitudes and are reported in centimeters. From [90]

\subsubsection{DR of the lung}

From end-exhale to end-inhale. A first group of studies have explored DR between EEX and EIN CT images acquired during breath hold. Several groups used sparse sets of anatomical features (mostly selected points or extracted surfaces) in each image, established pairs of correspondence between features and finally computed dense displacement fields with an interpolation model based on the paired features $[94,20,21,77,28,95,76]$. In $[94,20,28]$, features were points manually located and paired by experts. In [95], features were automatically determined with the image gradient and, in [76], using slice by slice segmentation. Warping models used to compute dense displacement fields were: TPS $[28,21]$ with inverse-consistent constraints in [21], radial basis interpolation with the shift log function [76], and B-spline [95].

Other groups used dense intensity-based methods. Most authors [20,96,21,25,97,98] used SSD as dissimilarity measure and neglected the lung density variations due to 
breathing. Sundaram et al. [27] used normalized cross-correlation on 2D MRI slices, Coselmon [28] used mutual information on right lung images, Weruaga et al. [96] computed a similarity measure which was a combination of cross-correlation and SSD. We [26] proposed a preprocessing step, called a priori lung density modification (APLDM), to take into account changes in lung density due to inspiration.

4D Models. Attempts at building a 4D model have been made. A 4D model encompassed successive step-by-step deformations from EEX to EEI, and is not based on a single deformation from initial to final state. It contains individual voxel trajectories and could be used to study local hysteresis. A 4D model is generally (but not necessarily) build with from 4D CT images. 4D CT imaging [99] can be defined as the acquisition of a sequence of 3D CT image sets over consecutive segments of a breathing cycle. $4 \mathrm{D}$ acquisition is limited by the speed of the detectors and the gantry, and by the amount of delivered dose. Several CT acquisition modes were used: cine mode [100], helical mode [101], on-board Cone-Beam [102] or dedicated 4D CT (256-slices) [103,104]. 4D reconstruction is based on a synchronized respiratory signal acquired during data acquisition. Synchronization can depend on an external signal (surface motion tracking, with RPM, Real-Time Position Management from Varian $[105,106,107])$ or on a lung volume signal (spirometer $[108,109]$ ), or on an internal signal (tracking by image processing techniques $[102,110]$ ). The $4 \mathrm{D}$ dataset is then composed of about ten time-successive 3D images reconstructed from these datasets.

Zhang et al. [57] proposed to build a 4D thorax model with a triangular surface mesh obtained from lung contours converted into a volumetric tetrahedral mesh(see fig.15). Transformation from the EEX image to the EIN image was then computed with a finite element contact-impact analysis allowing to simulate interactions between the organ of interest and the surrounding body. As described before, Brock et al. [92] used their finite element model-based multi-organ deformable image registration on the thoracic region including the lungs, the external surface and the breasts. Rietzel et al. [111] used an open source implementation of an intensitybased free-form DR based on B-spline interpolation [112]. The similarity measure was SSD. DF was used to combine dose distributions and a technique based on maximum intensity volume of lung tumor was proposed for fast contouring of composite GTV. Kaus et al [98] compared deformable surface (based on manually drawn 2D contours) and volumetric B-spline DR to propagate organ models through the image time series. They concluded that surface DR is not able to handle tumor motion, but that intensity-based DR was susceptible to motion artifacts in CT. Keall et al. [113] used large deformation diffeomorphic image registration from $[40,114]$ to map the transformation between a reference $\mathrm{CT}$ image (peak-inhale) to any other $\mathrm{CT}$ image in the $4 \mathrm{D}$ dataset. The resulting DF allows the contours defined on one image to be automatically transferred to other phases images (eight image sets in total), thus allowing to draw 4D contours. Guerrero et al. [115] proposed another use of DR in 4D CT images: they described a method of quantifying regional ventilation with the goal of developing functional images for treatment planning and optimization. Optical-flow DR [97] was used to obtain voxel to voxel correspondence, and local volume change due to inspiration was computed using corresponding Hounsfield units. A similar approach was also used by our team [26] to generate intermediate 
voxel densities taking into account the air volume change, for simulating a 4D image from two EEX-EIN breath hold CT scans.
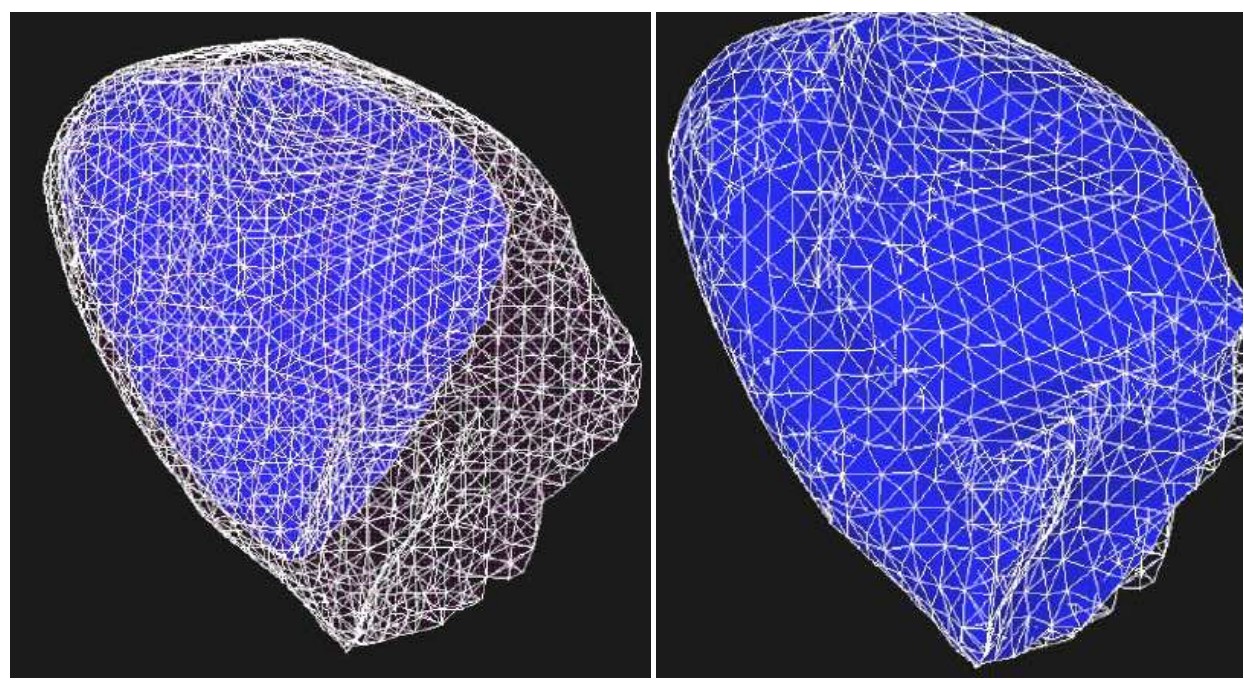

Fig. 15. The initial state (left) and ending state (right) of finite element contact-impact analysis. The transparent mesh is the contact target surface extracted from the images of the lung at inhalation phase; the inner mesh is the solid lung model created from the exhalation lung images. After simulation, the solid lung model occupied the space enclosed by the surface of the lung at inhalation phase. From $[57]$
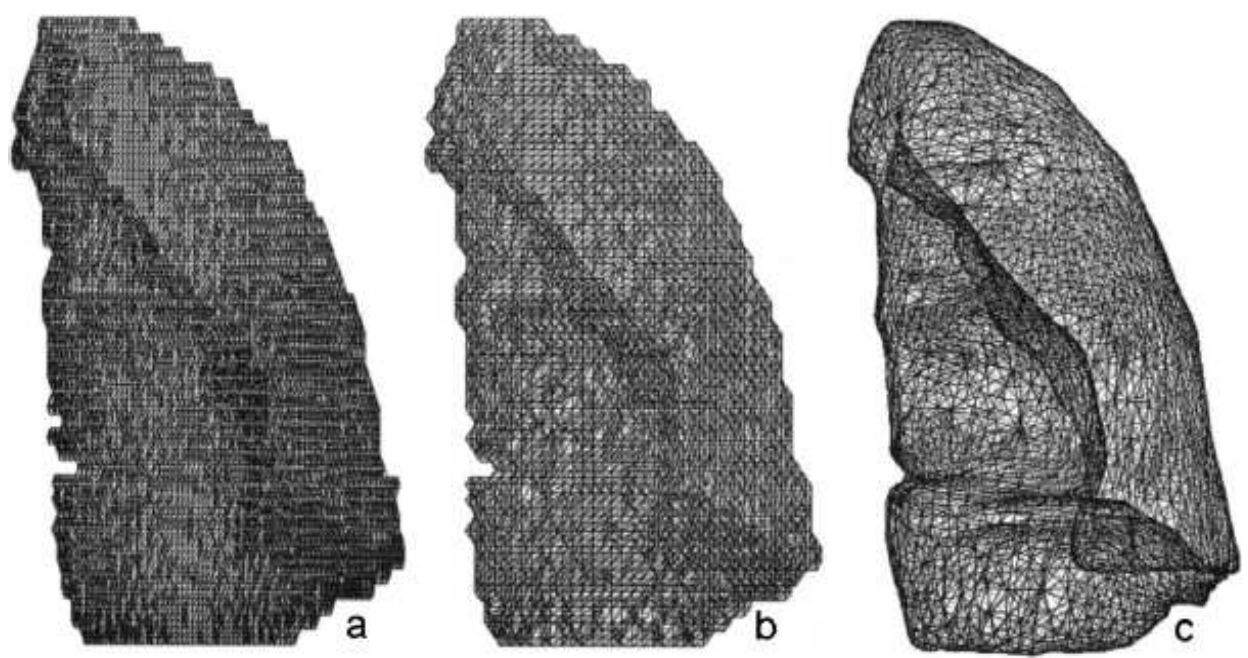

Fig. 16. Smoothing process for lungs: (left) original mesh with no data reduction and no smoothing, (middle) reduced to $128 \times 128$ data, (right) reduced to $128 \times 128$, decimated and smoothed. From [92]

\subsubsection{Conclusion}

A spatio-temporal 4D model allows to quantify and analyze the displacement and deformation of any object (organ, tumor) as a function of time. Variations of lung density according to time can be computed. Any (ir)regular breathing signal can 
be simulated. When related to a given breathing signal, Probability Density Function (PDF) of organ/tumor presence can be computed in order to derive optimal treatment margins. The $4 \mathrm{D}$ model can be incorporated into the treatment plan by dose deformation as in [116] or combination of dose distributions [111,91] for lung or liver. Keall et al. [113] used such a model to perform 4D dose computation for DMLC-based (Dynamic Multi-Leaf Collimator) respiratory motion tracking. Several authors used 4D models with Monte-Carlo simulations $[117,118,119]$. Rit et al. [110] simulated dynamic cone-beam image acquisitions. Resulting 4D deformation fields were also used to propagate contours from one image to the other in a $4 \mathrm{D}$ dataset $[111,113]$.

However, such 4D models are still in their early stages. They are subject to image artifacts, do not take into account heart beat variability [120], or respiration deficiencies (cirrhosis, atelectasis, emphysema) and still need validation. Nevertheless, $4 \mathrm{D}$ DR are very promising tools that provide individualized quantitative information for each patient on organ trajectories or hysteresis, for instance.

\subsection{Example $n^{\circ} 4:$ PET (motion compensation)}

${ }^{18} \mathrm{~F}$ fluorodioxydoglucose (FDG) Positron emission tomography (PET) images provide useful functional information which could lead to improve diagnosis or perform PET-based radiotherapy planning. However, registering these images to anatomical $\mathrm{CT}$ is difficult due to the acquisition difference between the two modalities: the process requires a few seconds acquisition time for CT, about 30 min for PET; motion artifacts caused by respiration thus degrade PET image quality and quantification. Moreover, differences in the patient positions (arms-up posture) cause RR failure, with two major consequences: it affects the accuracy of quantification, producing a reduction of the measured standard uptake value (SUV) and the apparent lesion volume is overestimated [121].

Slomka et al. [122] proposed a fully automated algorithm for DR of whole-body PET and $\mathrm{CT}$ images, which compensates for the nonlinear deformation due to breathhold CT imaging. After initial affine registration based on mutual information, they used TPS on a large number (a few hundreds) of automatically derived corresponding point pairs. Shekhar et al. [123] used DR to register and fuse a whole-body functional PET with an anatomic CT, in order to differentiate viable tumors from benign masses. They used elastic intensity-based DR with normalized mutual information, and the global transformation was derived from a combination of multiple local rigid body transformations. Mattes et al.[124] proposed a method to elastically align PET (normal tidal during about 30 min, without arms-up posture) and CT images (acquired in DI breath-hold, with arms-up posture) of the chest. They used mutual information measure with B-spline based DR (both for deformation parameterization and image value interpolation), and limited-memory quasi-Newton optimization algorithm. Schwartz et al. [125] used this technique for head and neck PET-CT imaging in order to guide head and neck intensity-modulated radiation therapy planning [126] and to improve neck staging sensitivity and specificity in pa- 
tients with head and neck squamous cell cancer [125]. Reyes et al. [127] proposed a statistical method to correct for PET motion artifacts based on a 4D motion model. Such technique require the a priori knowledge of a $4 \mathrm{D}$ motion model such as the ones described in the previous section.

Nehmeh et al. $[121,128,129]$ proposed a protocol to acquire gated 4D-PET/CT images whereas circumventing breathing motion artifacts in cases where DR could potentially be used. Once the images are registered, the resulting DF can be applied to the emission or standard uptake value image for building a fused PET-CT image and improving PET image interpretation.

\subsection{Example $n^{\circ}$ 5: Inter-patient registration (atlas)}

Intra-patient DR is generally used to account for the deformation of anatomical structures corresponding to a "real", elastically or plastically deforming material. Inter-patient DR is used to help transferring contours from one patient in another and/or to study the variability of anatomical structures across different patients. Generally, (semi-)automated organ delineation with inter-patient DR is performed based on an anatomical atlas, composed of several images which are segmented and represented in a same reference frame system.

Qatarneh et al. [130] constructed a whole body atlas for optimizing radiotherapy. The atlas contained, among other information, standardized, segmented organs from several patients, to be used as a reference. Auto-segmentation was then performed for each individual patient, first by matching contours from to whole body atlas to the new image, then by refining contours using an active contour model. Boes et al. [131] generated a normalized geometric liver model using DR with TPS surface warping. Li et al. [21] built a normative atlas of the human lung for inter-patient thorax images. Bondiau et al. [132] used an intensity-based DR technique (SSD, fluid, optical flow) to register brain MRI to another segmented MRI, allowing automatic delineation of brain structures. Park et al. [133] proposed a method to construct a probabilistic atlas of an abdomen consisting of four organs: liver, kidneys, and spinal cord. They used 32 manually segmented CT images mapped into a same reference with a quasi-fully automated DR (using TPS and mutual information). The probabilistic atlas was used to aid the segmentation of low-contrast organs. Deurloo et al. [71] used a morphing technique [134] to map manually drawn GTVs (prostate and seminal vesicles) of several patients to a reference case. Although the technique used (morphing of 3D surfaces) is not strictly a DR, it detects deformations between GTV's, allowing statistical comparisons.

DF amplitudes are generally higher with inter-patient DR than with intra-patient DR and thus potentially require a higher number of degrees of freedom to model the deformation, and more iterations to converge. However, regularization is generally less constrained than with intra-patient DR. More details on inter-subject DR can be found in [135]. 


\section{Conclusion}

Sarrut. 24

Many other examples of DR use in radiotherapy can be found. Christensen et al. [114] used fluid intensity-based DR between cervix CT images for planning brachytherapy treatments with intracavitary applicators (which induce complex organ deformations). Our group [136] evaluated inter-fraction reproducibility by comparing 3D CT images acquired from the same patient at the same breath-hold levels. Bharatha et al. [137] proposed to recover shape changes between pre-operative MRI images obtained with an endorectal coil and intra-operative images obtained with a rectal obturator in place. The method involved the use of a biomechanical FEM (with tetrahedral elements) initially obtained from segmented images $[138,139]$.

The main difficulty is validation. There is no standardized means of evaluating the results of a DR method. The most common evaluation methods are:

- Simulated data: this method involves the generation of an artificial image deformation with a mathematical transformation. Its advantage is that the gold standard is perfectly known. However, these method lacks realism. It is generally only a first step and allows to evaluate the influence of parameters (noise quantity) on the method. Evaluating the consistence of the deformation (Invertibility: is $\phi_{i j}$ close to $\phi_{j i}$ ? Transitivity: is $\phi_{i j} \circ \phi_{j k}$ close to $\phi_{i k}$ ?) could also provide useful information $[48,140]$.

- Phantom data: the method is more realistic but still uncertain. The gold standard is evaluated by means of implanted landmarks. For example, Wang et al. [70] designed a deformable pelvis phantom containing simulated prostate, bladder, seminal vesicles, rectum and bony structures. A rectal balloon could be inflated and seeds were embedded in the prostate structure in order to track the deformation.

- Patient data: the gold standard is unknown. It can be defined by means of manual selection of homologous landmarks by experts. However, even if the found transformation perfectly matches the expert-defined landmarks, this does not imply that the transformation of points between the landmarks is correct.

Even if the validation of DR is still uncertain, some applications can benefit from these techniques. For the propagation of organs/tumor contours, for instance, the distance between deformed contours and those defined by experts should remain in the range of inter-experts variability. However, for more precise applications such as dose deformation, or organ deformation studies, the results of DR algorithms should be used with care. Nevertheless, DF algorithms remain a fundamental image analysis tools for radiotherapy, and will probably be included into all treatment planning systems in the near future. 


\section{A Equations for transformation models}

\section{A.1 Global polynomial transformation}

$$
\phi(\mathbf{x})=\sum_{i j k}^{m} \alpha_{i j k} x_{1}^{i} x_{2}^{j} x_{3}^{k}=\mathbf{P}^{m}(\mathbf{x})
$$

where $\alpha$ is the three-component vector of coefficients for the three components of $\mathbf{x}=\left(x_{1}, x_{2}, x_{3}\right) \cdot m$ is the order of the polynomial. We obtain $n=\frac{(d+m) !}{(d ! m !)}$ parameters for each dimension. For example, quadratic (second order) polynomials $(m=2)$ for 3D images $(d=3)$ lead to $n=10$ parameters for each component of $\mathbf{x}^{\prime}, \boldsymbol{\alpha}$ is thus a $10 \times 3$ matrix with 30 degrees of freedom. Cubic, fourth and fifth order polynomials lead to 60, 105 and 168 degrees of freedom.

\section{A.2 Local nonrigid transformations: piecewise polynomials}

\section{A.2.1 Non-compact support splines}

Interpolation with a general radial basis function approach of $n$ pairs of landmarks $\left(\mathbf{p}_{i}, \mathbf{q}_{i}\right)$ is:

$$
\begin{aligned}
\phi\left(\mathbf{p}_{i}\right) & =\mathbf{q}_{i} \quad \forall i \\
\phi(\mathbf{x}) & =\mathbf{P}^{m}(\mathbf{x})+\sum_{i}^{n} c_{i} R\left(\left\|\mathbf{x}-\mathbf{p}_{i}\right\|\right)
\end{aligned}
$$

with $\mathbf{P}^{m}$ the sum of polynomials up to order $m$ (see eq. A.1). $R$ is a function depending only on the distance $r \geq 0$ from the origin $\left\|\mathbf{x}-\mathbf{p}_{i}\right\|=\|r\|$ (radial basis). $c_{i}$ stands for the coefficients to be found. Commonly used RBF are thin-plate spline, multiquadrics, inverse multiquadrics and Gaussians:

$$
\begin{aligned}
R_{T P S}(r) & = \begin{cases}r^{4-d} \ln r, & 4-d \in 2 \mathbb{N} \\
r^{4-d}, & \text { otherwise. }\end{cases} \\
R_{M}(r) & =\left(r^{2}+c^{2}\right)^{\mu}, \quad \mu \in \mathbb{R}^{+} \\
R_{I M}(r) & =\left(r^{2}+c^{2}\right)^{-\mu}, \quad \mu \in \mathbb{R}^{+} \\
R_{G}(r) & =\exp ^{-r^{2} / 2 \sigma^{2}}
\end{aligned}
$$

Others examples of RBF with compact support are found in [39] with $\psi$-functions 
of Wendland and [141] with truncated Gaussians.

\section{A.2.2 B-splines}

Deformation is represented as a linear combination of uniformly-spaced cubic Bsplines $[30,75]$ :

$$
\begin{aligned}
\phi(\mathbf{x}) & =\sum_{l=0}^{r} \sum_{m=0}^{r} \sum_{n=0}^{r} \mathbf{c}_{i+l, j+m, k+n} B_{l}^{r}(u) B_{m}^{r}(v) B_{n}^{r}(w) \\
u & =x_{1} / \delta_{1}-(i+1) \quad i=\left\lfloor x_{1} / \delta_{1}\right\rfloor-1 \\
v & =x_{2} / \delta_{2}-(j+1) \quad j=\left\lfloor x_{2} / \delta_{2}\right\rfloor-1 \\
w & =x_{3} / \delta_{3}-(k+1) \quad k=\left\lfloor x_{3} / \delta_{3}\right\rfloor-1 \\
B_{0}^{3}(a) & =\left(-a^{3}+3 a^{2}+3 a+1\right) / 6 \\
B_{1}^{3}(a) & =\left(3 a^{3}-6 a^{2}+4\right) / 6 \\
B_{2}^{3}(a) & =\left(-3 a^{3}+3 a^{2}+3 a+1\right) / 6 \\
B_{3}^{3}(a) & =a^{3} / 6
\end{aligned}
$$

where $\mathbf{c}$ are a lattice of uniformly spaced control points with spacing $\delta_{1} \times \delta_{2} \times \delta_{3}$, and $B_{i}$ the $i^{\text {th }}$ basis function of the B-spline. Such formulation stores the absolute control points positions and not their relative displacements. Alternatively, Kybic et al [34] parameterized the B-spline as follows (leading to the same results):

$$
\begin{aligned}
\phi(\mathbf{x}) & =\mathbf{x}+\sum_{\mathbf{k} \in \mathbf{K}} \mathbf{c}_{\mathbf{k}} \beta^{r}\left(x_{1} / h_{1}-k_{1}\right) \beta^{r}\left(x_{2} / h_{2}-k_{2}\right) \beta^{r}\left(x_{3} / h_{3}-k_{3}\right) \\
\beta^{r} & =\beta^{r-1} * \beta^{0} \text { for } r>0, \quad \beta^{0}(a)= \begin{cases}1 & \text { if } \quad a \in\left(-\frac{1}{2}, \frac{1}{2}\right) \\
0 & \text { otherwise }\end{cases} \\
\beta^{3}(a) & = \begin{cases}2 / 3-(1-|a| / 2) a^{2} & \text { if } 0<|a| \leq 1 \\
(2-|a|)^{3} / 6 & \text { if } 1<|a|<2 \\
0 & \text { otherwise }\end{cases}
\end{aligned}
$$

where $\mathbf{K}=\left\{\mathbf{k}=\left(k_{1}, k_{2}, k_{3}\right)\right\}$ is the set of control points, $r$ is the degree of splines used and $\mathbf{h}=\left(h_{1}, h_{2}, h_{3}\right)$ the regular spacing of control points. If the node spacing variables $\mathbf{h}$ are required to be integer, the values of the B-spline $\beta^{r}$ can be precalculated with a small number of points and a given displacement $\phi(\mathbf{x})$ can be evaluated with $3(r+1)$ multiplications. 


\section{A.2.3 Some regularization energies}

Let $\phi(\mathbf{x})=\mathbf{x}+\mathbf{u}(\mathbf{x})$ be the deformation field, with $\mathbf{u}=\left(u_{1}, u_{2}, u_{3}\right)$. In the following, $\Omega$ denotes an image domain. In each equation, $(\mathbf{x})$ is omitted in expressions like $\mathbf{u}(\mathbf{x})$ or $u_{i}(\mathbf{x})$. The following equations correspond to some of the regularization energies $E$ used for DR:

- Frobenius's norm [25,97] (defined as the square root of the sum of the absolute squares of its elements; the square root is often omitted):

$$
E_{F}(\mathbf{u})=\int_{\mathbf{x} \in \Omega}\|\nabla \mathbf{u}\|^{2} d \mathbf{x}=\int_{\mathbf{x} \in \Omega} \sqrt{\sum_{k=1}^{3} \sum_{l=1}^{3}\left|\frac{\partial u_{k}}{\partial x_{l}}\right|^{2}} d \mathbf{x}
$$

- Laplacian or membrane energy [41]:

$$
\begin{aligned}
E_{m}(\mathbf{u}) & =\int_{\mathbf{x} \in \Omega}\|\Delta \mathbf{u}\|^{2} d \mathbf{x}=\int_{\mathbf{x} \in \Omega}\left\|\nabla^{2} \mathbf{u}\right\|^{2} d \mathbf{x} \\
& =\int_{\mathbf{x} \in \Omega}\left\|\sum_{i=1}^{3} \frac{\partial^{2} \mathbf{u}}{\partial x_{i}^{2}}\right\|^{2} d \mathbf{x}=\int_{\mathbf{x} \in \Omega}\left[\begin{array}{l}
\sum_{i=1}^{3} \frac{\partial^{2} u_{1}}{\partial x_{i}^{2}} \\
\sum_{i=1}^{3} \frac{\partial^{2} u_{2}}{\partial x_{i}^{2}} \\
\sum_{i=1}^{3} \frac{\partial^{2} u_{3}}{\partial x_{i}^{2}}
\end{array}\right]^{2} d \mathbf{x}
\end{aligned}
$$

- Biharmonic energy (thin-plate, minimum bending energy) $[47,46]$ :

$$
E_{t p s}(\mathbf{u})=\int_{\mathbf{x} \in \Omega} \Delta^{2} \mathbf{u} d \mathbf{x}=\int_{\mathbf{x} \in \Omega} \nabla^{4} \mathbf{u} d \mathbf{x}=\int_{\mathbf{x} \in \Omega} \sum_{i=1}^{3} \sum_{j=1}^{3}\left(\frac{\partial u_{i}^{2}}{\partial x_{i} \partial x_{j}}\right)^{2} d \mathbf{x}
$$

- Linear elastic energy [40], with $\lambda$ and $\mu$ the Lamé constants (which are related to the Young's modulus $E$ and Poisson's ratio):

$$
E_{L E}(\mathbf{u})=\int_{\mathbf{x} \in \Omega} \frac{\lambda}{2}(\operatorname{div}(\mathbf{u}))^{2}+\mu\|\nabla \mathbf{u}\|^{2}-\frac{\mu}{2}\|\operatorname{rot}(\mathbf{u})\|^{2} d \mathbf{x}
$$

Poisson's ratio Young's modulus

$$
\nu=\frac{\lambda}{2(\lambda+\mu)} \quad E=\frac{\mu(3 \lambda+2 \mu)}{\lambda+\mu}
$$

- Jacobian-related energy [75,34]:

$$
\begin{aligned}
& E_{J 1}(\mathbf{u})=\int_{\mathbf{x} \in \Omega}|\log (\operatorname{det} \nabla \mathbf{u})| d \mathbf{x} \\
& E_{J 2}(\mathbf{u})=\int_{\mathbf{x} \in \Omega} \exp ^{-\alpha \operatorname{det} \nabla \mathbf{u}} d \mathbf{x}
\end{aligned}
$$




\section{References}

[1] Jaffray D. Emergent technologies for 3-dimensional image-guided radiation delivery. Semin Radiat Oncol, 15(3):208-16 2005.

[2] Yan D, Lockman D, Martinez A, Wong J, Brabbins D, Vicini F, Liang J and L K. Computed tomography guided management of interfractional patient variation. Semin Radiat Oncol, 15(3):168-79 2005.

[3] Shiu A, Chang E, Ye J, Lii M, Rhines L, Mendel E, Weinberg J, Singh S, Maor M, Mohan R and Cox J. Near simultaneous computed tomography imageguided stereotactic spinal radiotherapy: an emerging paradigm for achieving true stereotaxy. Int J Radiat Oncol Biol Phys, 57(3):605-13 2003.

[4] Jaffray D, Siewerdsen J, Wong J and Martinez A. Flat-Panel Cone-Beam Computed Tomography for Image-Guided Radiation Therapy. Int $J$ Radiat Oncol Biol Phys, 53(5):1337-1349 2002.

[5] Ford E, Chang J, Mueller K, Sidhu K, Todor D, Mageras G, Yorke E, Ling $\mathrm{C}$ and Amols H. Cone-beam CT with megavoltage beams and an amorphous silicon electronic portal imaging device: potential for verification of radiotherapy of lung cancer. Med Phys, 29(12):2913-24 2002.

[6] Cabral B and Leedom L. Imaging Vector Fields Using Line Integral Convolution. In International Conference on Computer Graphics and Interactive Techniques, SIGGRAPH, pages 263-270. ACM Press New York, NY, USA 1993.

[7] Post F, Vrolijk B, Hauser H, Laramee R and Doleisch H. The State of the Art in Flow Visualisation: Feature Extraction and Tracking. Computer Graphics Forum, 22(4):775-92 2003.

[8] Laramee R, Hauser H, Doleisch H, Vrolijk B, Post F and Weiskopf D. The State of the Art in Flow Visualization: Dense and Texture-Based Techniques. Computer Graphics Forum, 23(2):203-221 2004.

[9] Crum W, Hartkens T and Hill D. Non-rigid image registration: theory and practice. Br J Radiol., 77(2):140-153 2004.

[10] Zitova B and Flusser J. Image registration methods: a survey. Im. Vis. Comp., 21:977-1000 2003.

[11] Pluim J, Maintz J and Viergever M. Mutual information based registration of medical images: a survey. IEEE Trans Med Imaging, 22(8):986-1004 2003.

[12] Dawant B. Non-rigid registration of medical images: purpose and methods, a short survey. In IEEE International Symposium onBiomedical Imaging, pages 465-68. Washington DC, USA 2002.

[13] Rueckert D. Medical Image Registration, chapter (13) Non-rigid registration: Techniques and applications, pages 281-303. CRC Press 2001. 
[14] Hill D, Batchelor P, Holden M and Hawkes D. Medical image registration. Phys Med Biol, 46(3):R1-45 2001.

[15] Fitzpatrick J, Hill D and Maurer Jr C. Image registration ; Handbook of Medical Imaging, volume 2 of Medical Image Processing and Analysis, chapter 8. SPIE Press, Bellingham, Wash, m. sonka and j.m. fitzpatrick edition 2000.

[16] Audette M, Ferrie F and Peters T. An algorithmic overview of surface registration techniques for medical imaging. Med Image Anal, 4:201-217 2000.

[17] Rohr K. Elastic Registration of Multimodal Medical Images: A Survey. Künstliche Intelligenz, pages 11-17 2000.

[18] Lester H and Arridge S. A survey of hierarchical non-linear medical image registration. Pat Recog, 32(1):129-149 1999.

[19] Maintz J and Viergever M. A survey of medical image registration. Medical Image Analysis, 2(1):1-36 1998.

[20] Fan L, Chen C, Reinhardt J and Hoffman E. Evaluation and application of 3D lung warping and registration model using HRCT images. In SPIE Medical Imaging, volume 4321, pages 234-243. San Diego, CA 2001.

[21] Li B, Christensen G, Hoffman E, McLennan G and Reinhardt J. Establishing a normative atlas of the human lung: intersubject warping and registration of volumetric CT images. Acad. Radiol., 10(3):255-265 2003.

[22] Yan D, Jaffray D and Wong J. A model to accumulate fractionated dose in a deforming organ. Int J Radiat Oncol Biol Phys, 44(3):665-75 1999.

[23] Schaly B, Kempe J, Bauman G, Battista J and Van Dyk J. Tracking the dose distribution in radiation therapy by accounting for variable anatomy. Phys Med Biol, 49(5):791-805 2004.

[24] Rohr K. Extraction of 3D Anatomical Point Landmarks Based on Invariance Principles. Pat Recog, 32:3-15 1999.

[25] Lu W, Chen M, Olivera G, Ruchala K and Mackie T. Fast free-form deformable registration via calculus of variations. Phys Med Biol, 49(14):3067-3087 2004.

[26] Sarrut D, Boldea V, Miguet S and Ginestet C. Simulation of 4D CT images from deformable registration between inhale and exhale breath-hold CT scans. Med Phys, 33(3):605-617 2006.

[27] Sundaram T and Gee J. Towards a model of lung biomechanics: pulmonary kinematics via registration of serial lung images. Med Image Anal, 9(6):254-37 2005.

[28] Coselmon M, Balter J, McShan D and Kessler M. Mutual information based CT registration of the lung at exhale and inhale breathing states using thinplate splines. Med Phys, 31(11):2942-2948 2004. 
[29] Brock K, Balter J, Dawson L, Kessler M and Meyer C. Automated generation of a four-dimensional model of the liver using warping and mutual information. Med Phys, 30(6):1128-33 2003.

[30] Rueckert D, Sonoda L, Hayes C, Hill D, Leach M and Hawkes D. Nonrigid, registration using free-form deformations: Application to breast MR images. IEEE Trans Med Imaging, 18(8):712-721 1999.

[31] Roche A, Malandain G and Ayache N. Unifying Maximum Likelihood Approaches in Medical Image Registration. Int J of Comput Vision, 11(1):7180 2000. Special Issue on 3D Imaging.

[32] Hermosillo G, Chefd'hotel C and Faugeras O. Variational methods for multimodal image matching. Int J of Comput Vision, 50(3):329-43 2002.

[33] Johnson $\mathrm{H}$ and Christensen G. Consistent Landmark and Intensity-based Image Registration. IEEE Trans Med Imaging, 21(5):450-61 2002.

[34] Kybic J and Unser M. Fast Parametric Elastic Image Registration. IEEE T Im Proc, 12(11):1427-42 2003.

[35] Woods R, Grafton S, Watson J, Sicotte N, and Mazziotta J. Automated image registration: II. intersubject validation of linear and nonlinear models. J Comput Assist Tomogr, 22(1):153-165 1998.

[36] Wu X, Dibiase S, Gullapalli R and Yu C. Deformable image registration for the use of magnetic resonance spectroscopy in prostate treatment planning. Int J Radiat Oncol Biol Phys, 58(5):1577-1583 2004.

[37] Ashburner J and Friston K. Nonlinear spatial normalization using basis functions. Hum Brain Mapp, 7(4):254-66 1999.

[38] Bookstein F. Principal warps: Thin-plate splines and the decomposition of deformations. IEEE T Pat An Mach Int, 11:567-585 1989.

[39] Fornefett M, Rohr K and Stiehl H. Radial Basis Functions with Compact Support for Elastic Registration of Medical Images. Im. Vis. Comp., 19(12):87-96 2001.

[40] Christensen G and Johnson H. Consistent image registration. IEEE Trans Med Imaging, 20(7):568-582 2001.

[41] Gee J, Reivich M and Bajscy R. Elastically deforming 3D atlas to match anatomical brain images. J Comput Assist Tomogr, 17:225-36 1993.

[42] Davis M, Khotanzad A, Flamig D and Harms S. Elastic Body Splines: A Physics Based Approach to Coordinate Transformation in Medical Image Matching. In Eighth IEEE Symposium on Computer-Based Medical Systems, page 81. IEEE Computer Society, Lubbock, TX, USA 1995.

[43] Chen F and Suter D. Image Coordinate Transformation Based on DIV-CURL Vector Splines. In IEEE Comp. Vis. Pat. Rec. (CVPR), volume 1, pages 518-521. IEEE Computer Society Press, Brisbane, Australia 1998. 
[44] Rohr K, Stiehl H, Sprengel R, Buzug T, Weese J and Kuhn M. LandmarkBased Elastic Registration Using Approximating Thin-Plate Splines. IEEE Trans Med Imaging, 20(6):526-34 2001.

[45] Bajscy R and Kovacic S. Multiresolution elastic matching. Computer Vision, Graphics and Image Processing, 46:1-21 1989.

[46] Christensen G, Rabbit R and Miller M. 3D brain mapping using a deformable neuroanatomy. Phys Med Biol, 39(3):609-618 1994.

[47] Bro-Nielsen M and Gramkow C. Fast Fluid Registration of Medical Images. In K Hone and R Kikinis, editors, SPIE Visualization in Biomedical Computing, volume 1131, pages 267-276. lncs 1996.

[48] Boldea V, Sarrut D and Carrie C. Comparison of 3D Dense Deformable Registration Methods for Breath-hold Reproducibility Study in Radiotherapy. In SPIE Medical Imaging: Visualization, Image-Guided Procedures, and Display, volume 5747, pages 222-230 2005.

[49] Boldea V, Sarrut D and Clippe S. Lung Deformation Estimation with NonRigid Registration for Radiotherapy Treatment. In MICCAI'2003, volume 2878, pages 770-7. LNCS 2003.

[50] Kybic J and Unser M. Multidimensional elastic registration of images using splines. In Int Conf Ima Proc (ICIP). Vancouver, Canada 2000.

[51] Rohlfing T and Jr C M. Intensity-based deformable, registration using adaptive multilevel free-form deformation with an incompressibility constraint. In W Niessen and M A Viergever, editors, MICCAI'2001, volume 2208, pages 111-119. LNCS, Utrecht (Netherlands) 2001.

[52] Thirion J. Image matching as a diffusion process: an analogy with Maxwell's demons. Med Image Anal, 2(3):243-260 1998.

[53] Cachier P and Ayache N. Isotropic energies, filters and splines for vectorial regularization. J Math Imaging Vis, 20(3):251-265 2004.

[54] Birkner M, Yan D, Alber M, Liang J and Nusslin F. Adapting inverse planning to patient and organ geometrical variation: algorithm and implementation. Med Phys, 30(10):2822-31 2003.

[55] Lian J, Xing L, Hunjan S, Dumoulin C, Levin J, Lo A, Watkins R, Rohling K, Giaquinto R, Kim D, Spielman D and Daniel B. Mapping of the prostate in endorectal coil-based MRI/MRSI and CT: a deformable registration and validation study. Med Phys, 31(11):3087-3094 2003.

[56] Chi Y, Liang J and Yan D. A material sensitivity study on the accuracy of deformable organ registration using linear biomechanical models. 33(2):4214332006 .

[57] Zhang T, Orton N, Mackie T and Paliwal B. Technical note: A novel boundary condition using contact elements for finite element based deformable image registration. Med Phys, 31(9):2412-5 2004. 
[58] Venugopal N, B M, Hnatov A and Dubey A. A feasibility study to investigate the use of thin-plate splines to account for prostate deformation. Phys Med Biol, 50(12):2871-85 2005.

[59] Lian J, Xing L, Hunjan S, Dumoulin C, Levin J, Lo A, Watkins R, Rohling K, Giaquinto R, Kim D, Spielman D and B D. Mapping of the prostate in endorectal coil-based MRI/MRSI and CT: a deformable registration and validation study. Med Phys, 31(11):3087-94 2004.

[60] Schreibmann E and Xing L. Narrow band deformable registration of prostate magnetic resonance imaging, magnetic resonance spectroscopic imaging, and computed tomography studies. Int J Radiat Oncol Biol Phys, 62(2):595-605 2005.

[61] Press W, Flannery B, Teukolsky S and Vetterling W. Numerical Recipes in C: The Art of Scientific Computing. Cambridge University Press, second edition 1992.

[62] Yan D, Vicini F, Wong J and Martinez A. Adaptive radiation therapy. Phys Med Biol, 42(1):12-32 1997.

[63] Langen $\mathrm{K}$ and Jones D. Organ motion and its management. Int $J$ Radiat Oncol Biol Phys, 50(1):265-78 2001.

[64] Wu C, Jeraj R, Olivera G and Mackie T. Re-optimization in adaptive radiotherapy. Phys Med Biol, 47(17):3181-95 2002.

[65] Wu C, Olivera G, Jeraj R, Keller H and TR M. Treatment plan modification using voxel-based weighting factors/dose prescription. Phys Med Biol, 48(15):2479-91 2003.

[66] Wu C, Jeraj R, Lu W and Mackie T. Fast treatment plan modification with an over-relaxed Cimmino algorithm. Med Phys, 31(2):191-200 2004.

[67] Schaly B, Bauman G, Battista J and Van Dyk J. Validation of contourdriven thin-plate splines for tracking fraction-to-fraction changes in anatomy and radiation therapy dose mapping. Phys Med Biol, 50(3):459-75 2005.

[68] Fei B, Duerk J, Sodee D and Wilson D. Semiautomatic nonrigid registration for the prostate and pelvic MR volumes. Acad. Radiol., 12(7):815-24 2005.

[69] Wang H, Dong L, Lii M, Lee A, de Crevoisier R, Mohan R, Cox J, Kuban D and Cheung R. Implementation and validation of a three-dimensional deformable registration algorithm for targeted prostate cancer radiotherapy. Int $J$ Radiat Oncol Biol Phys, 61(3):725-735 2005.

[70] Wang H, Dong L, O'Daniel J, Mohan R, Garden A, Ang K, Kuban D, Bonnen M, Chang J and Cheung R. Validation of an accelerated 'demons' algorithm for deformable image registration in radiation therapy. Phys Med Biol, 50(12):2887-905 2005. 
[71] Deurloo K, Steenbakkers R, Zijp L, de Bois JA, Nowak P, Rasch C and van Herk M. Quantification of shape variation of prostate and seminal vesicles during external beam radiotherapy. Int J Radiat Oncol Biol Phys, 61(1):228382005 .

[72] Kupelian P, Willoughby T, Meeks S, Forbes A, Wagner T, Maach M and Langen K. Intraprostatic fiducials for localization of the prostate gland: monitoring intermarker distances during radiation therapy to test for marker stability. Int J Radiat Oncol Biol Phys, 62(5):1291-6 2005.

[73] Smitsmans M, Wolthaus J, Artignan X, de Bois J, Jaffray D, Lebesque J and van Herk M. Automatic localization of the prostate for on-line or off-line image-guided radiotherapy. Int J Radiat Oncol Biol Phys, 60(2):623-35 2004.

[74] Schnabel J, Tanner C, AD C S, Degenhard A, Leach M, Hose D, DL H and Hawkes D. Validation of nonrigid image registration using finite-element methods: application to breast MR images. IEEE Trans Med Imaging, 22(2):238-47 2003.

[75] Rohlfing T, Maurer C J, Bluemke D and MA J. Volume-preserving nonrigid registration of MR breast images using free-form deformation with an incompressibility constraint. IEEE Trans Med Imaging, 22(6):730-41 2003.

[76] Matsopoulos G, Mouravliansky N, Asvestas P, Delibasis K and Kouloulias V. Thoracic deformable, registration combining self-organizing maps and radial basis functions. Med Image Anal, 9(3):237-254 2005.

[77] Betke M, Hong H, Thomas D, Prince C and Ko J. Landmark detection in the chest and registration of lung surfaces with an application to nodule registration. Med Image Anal, 7(3):265-281 2003.

[78] Jaffray D, Yan D and Wong J. Managing geometric uncertainty in conformal intensity-modulated radiation therapy. Semin Radiat Oncol, 9(1):4-19 1999.

[79] Schaly B, Bauman G, Song W, Battista J and Van Dyk J. Dosimetric impact of image-guided 3D conformal radiation therapy of prostate cancer. Phys Med Biol, 50(13):3083-101 2005.

[80] Mohan R, Zhang X, Wang H, Kang Y, Wang X, Liu H, Ang K, Kuban D and Dong L. Use of deformed intensity distributions for on-line modification of image-guided IMRT to account for interfractional anatomic changes. Int $J$ Radiat Oncol Biol Phys, 61(1):1258-66 2005.

[81] Yan D and Lockman D. Organ/patient geometric variation in external beam radiotherapy and its effects. Med Phys, 28(4):593-602 2001.

[82] Yan D, Xu B, Lockman D, Kota K, DS B, Wong J and Martinez A. The influence of interpatient and intrapatient rectum variation on external beam treatment of prostate cancer. Int J Radiat Oncol Biol Phys, 51(4):1111-9 2001.

[83] Ghilezan M, Yan D, Liang J, Jaffray D, Wong J and Martinez A. Online image-guided intensity-modulated radiotherapy for prostate cancer: How much 
improvement can we expect? A theoretical assessment of clinical benefits and potential dose escalation by improving precision and accuracy of radiation delivery. Int J Radiat Oncol Biol Phys, 60(5):1602-10 2004.

[84] Song W, Schaly B, Bauman G, Battista J and Van Dyk J. Image-guided adaptive radiation therapy (IGART): Radiobiological and dose escalation considerations for localized carcinoma of the prostate. Med Phys, 32(7):21932032005 .

[85] Goitein M. Organ and Tumor Motion: An Overview. Semin Radiat Oncol, 14(1):2-9 2004.

[86] Chen G, Kung J and Beaudette K. Artifacts in Computed Tomography Scanning of Moving Objects. Semin Radiat Oncol, 14(1):19-26 2004.

[87] Keall P, Mageras G, Balter J, Emery R, Forster K, Jiang S, Kapatoes J, Kubo H, Low D, Murphy M, Murray B, Ramsey C, Van Herk M, Sastry Vedam S, Wong J and Yorke E. The Management of Respiratory Motion in Radiation Oncology ; Handout for AAPM 2005 Continuing Education Session. Technical report, Report of AAPM Task Group 762005.

[88] Ling C, Yorke E, Amols H, Mechalakos J, Erdi Y, Leibel S, Rosenzweig K and Jackson A. Editorial : High-tech will improve radiotherapy of NSCLC: a hypothesis waiting to be validated. Int J Radiat Oncol Biol Phys, 60(1):3-7 2004 .

[89] Shirato H, Seppenwoolde Y, Kitamura K, Onimura R and S S. Intrafractional Tumor Motion: Lung and Liver. Semin Radiat Oncol, 14(1):10-18 2004.

[90] Brock K, Hollister S, Dawson L and Balter J. Technical note: creating a four-dimensional model of the liver using finite element analysis. Med Phys, 29(7):1403-5 2002.

[91] Brock K, McShan D, Ten Haken R, Hollister S, Dawson L and Balter J. Inclusion of organ deformation in dose calculations. Med Phys, 30(3):29052003.

[92] Brock K, Sharpe M, Dawson L, Kim S and Jaffray D. Accuracy of finite element model-based multi-organ deformable image registration. Med Phys, 32(6):1647-59 2005.

[93] Rohlfing T, Maurer Jr C, O'Dell W and Zhong J. Modeling liver motion and deformation during the respiratory cycle using intensity-based nonrigid registration of gated MR images. Med Phys, 31(3):427-432 2004.

[94] Fan L and Chen C. Integrated approach to 3D warping and registration from lung images. In U Bonse, editor, Proc. SPIE Developments in X-Ray Tomography II, volume 3772, pages 24-35 1999.

[95] Stewart C, Lee Y and Tsai C. An Uncertainty-Driven Hybrid of IntensityBased and Feature-Based Registration with Application to Retinal and Lung CT Images. In MICCAI'2004, volume 3217, pages 870-877. LNCS 2004. 
[96] Weruaga L, Morales J, Nunez L and Verdu R. Estimating Volumetric Motion in Thorax with Parametric Matching Constraints. IEEE Trans Med Imaging, 22(6):766-772 2003.

[97] Guerrero T, Zhang G, Huang T and Lin K. Intrathoracic tumour motion estimation from CT imaging using the 3D optical flow method. Phys Med Biol, 49:4147-4161 2004.

[98] Kaus M, Netsch T, Kabus S, Pekar V, McNutt T and Fischer B. Estimation of Organ Motion from 4D CT for 4D Radiation Therapy Planning of Lung Cancer. In MICCAI'2004, volume 3217, pages 1017-24. LNCS 2004.

[99] Keall P. 4-Dimensional Computed Tomography Imaging and Treatment Planning. Semin Radiat Oncol, 14(1):81-90 2004.

[100] Pan T. Comparison of helical and cine acquisitions for 4D-CT imaging with multislice CT. Med Phys, 32(2):627-634 2005.

[101] Keall P, Starkschall G, Shukla H, Forster K, Ortiz V, Stevens C, Vedam S, George R, Guerrero T and Mohan R. Acquiring 4D thoracic CT scans using a multislice helical method. Phys Med Biol, 49:2053-2067 2004.

[102] Sonke J, Zijp L, Remeijer P and van Herk M. Respiratory correlated cone beam CT. Med Phys, 32(4):1176-86 2005.

[103] Mori S, Endo M, Tsunoo T, Kandatsu S, Tanada S, Aradate H, Saito Y, Miyazaki H, Satoh K, Matsushita S and Kusakabe M. Physical Performance Evaluation of a 256 -Slice CT-Scanner for Four-Dimensional Imaging. Med Phys, 31(6):1348-1356 2004.

[104] Endo M, Tsunoo T, Kandatsu S, Tanada S, Aradate H and Saito Y. Four-dimensional computed tomography (4D CT)-concepts and preliminary development. Radiation Medicine, 21(1):17-22 2003.

[105] Rietzel E, Pan T and Chen G. Four-dimensional computed tomography: Image formation and clinical protocol. Med Phys, 32(4):974-889 2005.

[106] Pan T, Lee T, Rietzel E and Chen G. 4D-CT imaging of a volume influenced by respiratory motion on multi-slice CT. Med Phys, 31(2):333-340 2004.

[107] Vedam S, Keall P, Kini V, Mostafavi H, Shukla H and Mohan R. Acquiring a four-dimensional computed tomography dataset using an external respiratory signal. Phys Med Biol, 48(1):45-62 2003.

[108] Lu W, Parikh P, Naqa I E, Nystrom M, Hubenschmidt J, Wahab S, Mutic S, Singh A, anad JD Bradley G C and Low D. Quantization of the reconstruction quality of a four-dimensional computed tomography process for lung cancer patients. Med Phys, 32(4):890-901 2005.

[109] Low D, Nystrom M, Kalinin E, Parikh P, Dempsey J, Bradley J, Mutic S Wahab S, Islam T, Christensen G, Politte D and Whiting B. A method for the reconstruction of four-dimensional synchronized $\mathrm{CT}$ scans acquired during free breathing. Med Phys, 30(6):1254-63 2003. 
[110] Rit S, Sarrut D and Ginestet C. Respiratory signal extraction for 4D CT imaging of the thorax from cone-beam CT projections. In J Duncan and G Gerig, editors, MICCAI'2005, volume 3749, pages 556-63. LNCS 2005.

[111] Rietzel E, Chen G, Choi N and Willet C. Four-dimensional image-based treatment planning: Target volume segmentation and dose calculation in the presence of respiratory motion. Int $J$ Radiat Oncol Biol Phys, 61(5):1535-50 2005.

[112] Hartkens T, Rueckert D, Schnabel J, Hawkes D and Hill D. An open source software package for affine and non-rigid registration of single- and multimodal 3D images. In M Meiler, D Saupe, F Kruggel and et al, editors, Bildverarbeitung für die Medizin, Informatik aktuell, pages 409-12. SpringerVerlag 2002.

[113] Keall P, Joshi S, Vedam S, Siebers J, Kini V and Mohan R. Four-dimensional radiotherapy planning for DMLC-based respiratory motion tracking. Med Phys, 32(4):942-951 2005.

[114] Christensen G, Carlson B, Chao K, Yin P, Grigsby P, Nguyen K, Dempsey J, Lerma F, Bae K, Vannier M and Williamson J. Image-based dose planning of intracavitary brachytherapy: registration of serial-imaging studies using deformable anatomic templates. Int J Radiat Oncol Biol Phys, 51(1):227-43 2001.

[115] Guerrero T, Sanders K, Noyola-Martinez J, Castillo E, Zhang Y, Tapia R, Guerra R, Borghero Y and Komaki R. Quantification of regional ventilation from treatment planning CT. Int J Radiat Oncol Biol Phys, 62(3):630-4 2005.

[116] Zhang T, Jeraj R, Keller H, Lu W, Olivera G, McNutt T, Mackie T and Paliwal B. Treatment plan optimization incorporating respiratory motion. Med Phys, 31(6):1576-86 2004.

[117] Paganetti H. Four-dimensional Monte Carlo simulation of time-dependent geometries. Phys Med Biol, 49(6):N75-97 2004.

[118] Wang B, Goldstein M, Xu X and Sahoo N. Adjoint Monte Carlo method for prostate external photon beam treatment planning: an application to 3D patient anatomy. Phys Med Biol, 50(5):923-935 2005. Special Issue: International Workshop on current topics in Monte Carlo treatment planning.

[119] Keall P, Siebers J, Joshi S and Mohan R. Monte Carlo as a four-dimensional radiotherapy treatment-planning tool to account for respiratory motion. Phys Med Biol, 49(16):3639-3648 2004.

[120] Makela T, Clarysse P, Sipila O, Pauna N, Pham Q, Katila T and Magnin I. A review of cardiac image registration methods. IEEE Trans Med Imaging, 21(9):1011-21 2002.

[121] Nehmeh S, Erdi Y, Ling C, Rosenzweig K, Squire O, Braban L, Ford E, Sidhu K, Mageras G, Larson S and Humm J. Effect of respiratory gating on reducing lung motion artifacts in PET imaging of lung cancer. Med Phys, 29(3):366-71 2002. 
[122] Slomka P, Dey D, Przetak C, Aladl U and Baum R. Automated 3-dimensional registration of stand-alone (18)F-FDG whole-body PET with CT. J Nucl Med., 44(7):1156-67 2003.

[123] Shekhar R, Walimbe V, Raja S, Zagrodsky V, Kanvinde M, Wu G and Bybel B. Automated 3-dimensional elastic registration of whole-body PET and CT from separate or combined scanners. J Nucl Med., 46(9):1488-96 2005.

[124] Mattes D, Haynor D, Vesselle H, Lewellen T and Eubank W. PET-CT image registration in the chest using free-form deformations. IEEE Trans Med Imaging, 22(1):120-28 2003.

[125] Schwartz D, Ford E, Rajendran J, Yueh B, Coltrera M, Virgin J, Anzai Y, Haynor D, Lewellyn B, Mattes D, Meyer J, Phillips M, Leblanc M, Kinahan P, Krohn K, Eary J and Laramore G. FDG-PET/CT imaging for preradiotherapy staging of head-and-neck squamous cell carcinoma. Int J Radiat Oncol Biol Phys, 61(1):129-36 2005.

[126] Schwartz D, Ford E, Rajendran J, Yueh B, Coltrera M, Virgin J, Anzai Y, Haynor D, Lewellen B, Mattes D, Kinahan P, Meyer J, Phillips M, Leblanc M, Krohn K, Eary J and Laramore G. FDG-PET/CT-guided intensity modulated head and neck radiotherapy: a pilot investigation. Head Neck, 27(6):478-87 2005.

[127] Reyes M, Malandain G, Koulibaly P, Ballester M, and Darcourt J. Respiratory Motion Correction in Emission Tomography Image Reconstruction. In J Duncan and G Gerig, editors, MICCAI'2005, volume 3750, pages 369-76. LNCS 2004.

[128] Nehmeh S, Erdi Y, Pan T, Yorke E, Mageras G, Rosenzweig K, Schoder H, Mostafavi H, Squire O, Pevsner A, Larson S and Humm J. Quantitation of respiratory motion during 4D-PET/CT acquisition. Med Phys, 31(6):133313382004.

[129] Nehmeh S, Erdi Y, Pan T, Pevsner A, Rosenzweig K, Yorke E, Mageras G, Schoder H, Vernon P, Squire O, Mostafavi H, Larson S and Humm J. Fourdimensional (4D) PET/CT imaging of the thorax. Med Phys, 31(12):317931862004.

[130] Qatarneh S, Noz M, Hyodynmaa S, Maguire G J, Kramer E and Crafoord J. Evaluation of a segmentation procedure to delineate organs for use in construction of a radiation therapy planning atlas. Int J Med Inform, 69(1):39552003.

[131] Boes J, Bland P, Weymouth T, Quint L, Bookstein F, and Meyer C. Generating a normalized geometric liver model using warping. Invest. Radiol., 29:281-86 1994.

[132] Bondiau P, Malandain G, Chanalet S, Marcy P, Habrand J, Fauchon F, Paquis P, Courdi A, Commowick O, Rutten I and Ayache N. Atlas-based automatic segmentation of MR images: validation study on the brainstem in radiotherapy context. Int J Radiat Oncol Biol Phys, 61(1):289-98 2005. 
Sarrut. 38

[133] Park H, Bland P and Meyer C. Construction of an abdominal probabilistic atlas and its application in segmentation. IEEE Trans Med Imaging, 22(4):483-92 2003.

[134] DeCarlo D and Gallier J. Topological evolution of surfaces. In W A Davis, editor, Proc Graphics Interface, pages 194-203. Canadian Information Processing Society, Toronto, Ont., Canada 1996.

[135] Hellier P. Handbook of medical image analysis: segmentation and registration methods, volume 3, chapter Inter-subject non-rigid registration: survey and methods. Kluwer, j. suri and d. wilson and s. laxminarayan edition 2004.

[136] Sarrut D, Boldea V, Ayadi M, Badel J, Ginestet C and Clippe S. Non-rigid registration method to assess reproducibility of breath-holding with $\mathrm{ABC}$ in lung cancer. Int J Radiat Oncol Biol Phys, 61(2):594-607 2005.

[137] Bharatha A, Hirose M, Hata N, Warfield S, Ferrant M, Zou K, Suarez-Santana E, Ruiz-Alzola J, D'Amico A, Cormack R, Kikinis R, Jolesz F and Tempany C. Evaluation of three-dimensional finite element-based deformable registration of pre- and intraoperative prostate imaging. Med Phys, 28(12):2551-2560 2001.

[138] Ferrant M, Warfield S, Nabavi A, Macq B and Kikinis R. Registration of 3D intraoperative MR images of the brain using a finite element biomechanical model. In A DiGioia and S Delp, editors, MICCAI'2000, volume 1935, pages 19-28. lncs, Pittsburgh, USA 2000.

[139] Warfield S, Haker S, Talos I, Kemper C, Weisenfeld N, Mewes A, GoldbergZimring D, Zou K, Westin C, Wells W, Tempany C, Golby A, Black P, Jolesz $\mathrm{F}$ and Kikinis R. Capturing intraoperative deformations: research experience at Brigham and Women's Hospital. Med Image Anal, 9(2):145-62 2005.

[140] Jannin P, Fitzpatrick J, Hawkes D, Pennec X, R S and MW V. Validation of Medical Image Processing in Image-guided Therapy. In Comp. Assisted Radiology and Surgery (CARS). Elsevier Science 2002.

[141] Lotjonen J and Makela T. Elastic matching using a deformation sphere. In W Niessen and M A Viergever, editors, MICCAI'2001, volume 2208, pages 541-548. LNCS, Utrecht (Netherlands) 2001. 